\title{
Non-Abelian Seiberg-Witten Theory and stable oriented pairs
}

\author{
Andrei Teleman*
}

\section{Introduction}

The aim of this paper is to develop a systematic theory of non-abelian SeibergWitten equations. The equations we introduce and study are associated with a $\operatorname{Spin}^{G}(4)$-structure on a 4-manifold, where $G$ is a closed subgroup of the unitary group $U(V)$ containing the central involution $-\mathrm{id}_{V}$. We call these equations the $G$-monopole equations. For $G=S^{1}$, one recovers the classical (abelian) Seiberg-Witten equations [W], and the case $G=S p(1)$ corresponds to the "quaternionic monopole equations" introduced in [OT5]. Fixing the determinant of the connection component in the $U(2)$-monopole equations, one gets the so called $P U(2)$-monopole equations, which should be regarded as a twisted version of quaternionic monopole equations and will be extensively studied in the second part of this paper.

It is known ([OT4], [OT5], [PT2]) that the most natural way to prove the equivalence between Donaldson theory and Seiberg-Witten theory is to consider a suitable moduli space of non-abelian monopoles. In [OT5] it was shown that an $S^{1}$-quotient of a moduli space of quaternionic monopoles should give an homological equivalence between a fibration over a union of Seiberg-Witten moduli spaces and a fibration over certain Spin $^{c}$-moduli spaces [PT1].

By the same method, but using moduli spaces of $P U(2)$-monopoles instead of quaternionic monopoles, one should be able to express any Donaldson polynomial invariant in terms of Seiberg-Witten invariants associated with the twisted abelian monopole equations of [OT6].

The idea can be extended to get information about the Donaldson theories associated with an arbitrary symmetry group $G$, by relating the corresponding polynomial invariants to Seiberg-Witten-type invariants associated with smaller symmetry groups. One has only to consider a suitable moduli space of $G$ monopoles and to notice that this moduli space contains distinguished closed

\footnotetext{
*Partially supported by: AGE-Algebraic Geometry in Europe, contract No ERBCHRXCT940557 (BBW 93.0187), and by SNF, nr. 21-36111.92
} 
subspaces of "reducible solutions". The reducible solutions with trivial spinorcomponent can be identified with $G$-instantons, and all the others reductions can be regarded as monopoles associated to a smaller group.

It is important to point out that, if the base manifold is a Kähler surface one has Kobayashi-Hitchin-type correspondences (see [D], [DK], [K], [LT] for the instanton case) which give a complex geometric description of the moduli spaces of $S U(2), U(2)$ or $P U(2)$-monopoles (see section 2). The first two cases were already studied in [OT5] and [OT1]. In the algebraic case one can explicitly compute such moduli spaces of non-abelian monopoles and prove the existence of a projective compactification. The points corresponding to instantons and abelian monopoles can be easily identified (see also [OST]).

The theory has interesting extensions to manifolds of other dimensions. On Riemann surfaces for instance, one can use moduli spaces of $P U(2)$-monopoles to reduce the computation of the volume or the Chern numbers of a moduli space of semistable rank 2- bundles to computations on the symmetric powers of the base, which occur in the moduli space of $P U(2)$-monopoles as subspaces of abelian reductions.

The present paper is divided into two parts: The first deals with the general theory of $\operatorname{Spin}^{G}$-structures and $G$-monopole equations. We give classification theorems for $\operatorname{Spin}^{G}$-structures in principal bundles, and an explicit description of the set of equivalence classes in the cases $G=S U(2), U(2), P U(2)$. Afterwards we introduce the $G$-monopole equations in a natural way by coupling the Dirac harmonicity condition for a pair formed by a connection and a spinor, with the vanishing condition for a generalized moment map. This first part ends with a section dedicated to the concept of reducible solutions of the $G$-monopole equations. Describing the moduli spaces of $G$-monopoles around the reducible loci is the first step in order to express the Donaldson invariants associated with the symmetry group $G$ in terms of Seiberg-Witten-type reductions.

In the second part of the paper, we give a complex geometric interpretation of the moduli spaces of $P U(2)$-monopoles in terms of stable oriented pairs, by proving a Kobayashi-Hitchin type correspondence. Using this result, we describe a simple example of moduli space of $P U(2)$-monopoles on $\mathbb{P}^{2}$, which illustrates in a concrete case how our moduli spaces can be used to relate Donaldson and Seiberg-Witten invariants.

In order to be able to give general explicit formulas relating the Donaldson polynomial invariants to Seiberg-Witten invariants, it remains to construct $S^{1}$ equivariant smooth perturbations of the moduli spaces of $P U(2)$-monopoles, to construct an Uhlenbeck compactification of the perturbed moduli spaces, and finally to give explicit descriptions of the ends of the (perturbed) moduli spaces.

The first two problems are treated in [T1], [T2]. Note that the proof of the corresponding transversality results for other moduli spaces of non-abelian 
connections coupled with harmonic spinors ([PT1], [PT2]) are not complete ([T1]). The third problem, as well as generalizations to larger symmetry groups will be treated in a future paper.

I thank Prof. Christian Okonek for encouraging me to write this paper, as well as for the careful reading of the text and his valuable suggestions.

\section{G-Monopoles on 4-manifolds}

\subsection{The group $\operatorname{Spin}^{G}$ and $\operatorname{Spin}^{G}$-structures}

\subsection{1 $\operatorname{Spin}^{G}$-structures in principal bundles}

Let $G \subset U(V)$ be a closed subgroup of the unitary group of a Hermitian vector space $V$, suppose that $G$ contains the central involution $-\mathrm{id}_{V}$, and denote by $\mathfrak{g} \subset u(V)$ the Lie algebra of $G$. We put

$$
\operatorname{Spin}^{G}:=\operatorname{Spin} \times_{\mathbb{Z}_{2}} G .
$$

By definition we get the following fundamental exact sequences:

$$
\begin{gathered}
1 \longrightarrow \operatorname{Spin\longrightarrow } \operatorname{Spin}^{G} \stackrel{\delta}{\longrightarrow} G / \mathbb{Z}_{2} \longrightarrow 1 \\
1 \longrightarrow G \longrightarrow \operatorname{Spin}^{G} \stackrel{\pi}{\longrightarrow} S O \stackrel{\longrightarrow}{\longrightarrow} 1 \\
1 \longrightarrow \mathbb{Z}_{2} \longrightarrow \operatorname{Spin}^{G} \stackrel{(\pi, \delta)}{\longrightarrow} S O \times{ }^{G} / \mathbb{Z}_{2} \longrightarrow 1
\end{gathered}
$$

Note first that there are well defined morphisms

$$
\operatorname{ad}_{G}: \operatorname{Spin}^{G} \longrightarrow O(\mathfrak{g}), \operatorname{Ad}_{G}: \operatorname{Spin}^{G} \longrightarrow \operatorname{Aut}(G)
$$

induced by the morphisms ad $: G \longrightarrow O(\mathfrak{g})$ and Ad $: G \longrightarrow \operatorname{Aut}(G)$.

If $P^{G}$ is principal $S p i n^{G}$-bundle, we denote by $\mathbb{G}\left(P^{G}\right), \mathfrak{g}\left(P^{G}\right)$ the fibre bundles $P^{G} \times_{\mathrm{Ad}_{G}} G, P^{G} \times_{\operatorname{ad}_{G}} \mathfrak{g}$. The group of sections

$$
\mathcal{G}\left(P^{G}\right):=\Gamma\left(\mathbb{G}\left(P^{G}\right)\right)
$$

in $\mathbb{G}\left(P^{G}\right)$ can be identified with the group of bundle-automorphisms of $P^{G}$ over the $S O$-bundle $P^{G} \times_{\pi} S O$. After a suitable Sobolev completion $\mathcal{G}\left(P^{G}\right)$ becomes a Hilbert Lie group, whose Lie algebra is the corresponding Sobolev completion of $\Gamma\left(\mathfrak{g}\left(P^{G}\right)\right)$.

We put also

$$
\delta\left(P^{G}\right):=P^{G} \times{ }_{\delta}\left(G / \mathbb{Z}_{2}\right) .
$$

Note that $\mathbb{G}\left(P^{G}\right)$ can be identified with the bundle $\delta\left(P^{G}\right) \times{ }_{\overline{A d}} G$ associated with the $G / \mathbb{Z}_{2}$-bundle $\delta\left(P^{G}\right)$.

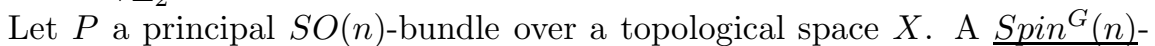
structure in $P$ is a bundle morphisms $P^{G} \longrightarrow P$ of type $\pi$, where $P^{G}$ is a 
principal $\operatorname{Spin}^{G}(n)$-bundle over $X$. Equivalently, a $\operatorname{Spin}^{G}(n)$-structure in $P$ can be regarded as a pair consisting of a $\operatorname{Spin}^{G}(n)$-bundle $P^{G}$ and an orientation preserving linear isometry

$$
\gamma: P \times_{S O(n)} \mathbb{R}^{n} \longrightarrow P^{G} \times_{\pi} \mathbb{R}^{n}
$$

(called the Clifford map of the structure).

Two Spin $^{G}$-structures $P_{0}^{G} \stackrel{\sigma_{0}}{\longrightarrow} P, P_{1}^{G} \stackrel{\sigma_{1}}{\longrightarrow} P$ in $P$ are called equivalent, if the $\operatorname{Spin}^{G}$ bundles $P_{0}^{G}, P_{1}^{G}$ are isomorphic over $P$.

If $(X, g)$ is an oriented Riemannian $n$-manifold, a $\operatorname{Spin}^{G}(n)$-structure in $X$ is a $\operatorname{Spin}^{G}(n)$-structure $P^{G} \longrightarrow P_{g}$ in the bundle $P_{g}$ of oriented $g$-orthonormal coframes of $X$. This is equivalent with the data of a pair $\left(P^{G}, \gamma\right)$, where $P^{G}$ is a. $\operatorname{Spin}^{G}(n)$-bundle and $\gamma: \Lambda_{X}^{1} \stackrel{\simeq}{\longrightarrow} P^{G} \times_{\pi} \mathbb{R}^{n}$ is a linear orientation-preserving isometry. Here $\Lambda_{X}^{1}$ stands for the cotangent bundle of $X$, endowed with the dual $S O(n)$-structure.

Let $X$ be a fixed paracompact topological space. Note that there is a natural map $H^{1}\left(X, G / \mathbb{Z}_{2}\right) \longrightarrow H^{2}\left(X, \mathbb{Z}_{2}\right)$, which we denote by $w$. If $G=\operatorname{Spin}(k), w$ coincides with the usual morphism $w_{2}$ defined on the set of $S O(k)$-bundles. By the third exact sequence in $(*)$ we get the following simple classification result

Proposition 1.1.1 The map $P^{G} \longmapsto\left(P^{G} \times_{\pi} S O, \delta\left(P^{G}\right)\right)$ defines a surjection of the set of isomorphism classes of Spin ${ }^{G}$-bundles onto the set of isomorphism classes of pairs $(P, \Delta)$ consisting of an SO-bundle and a $G / \mathbb{Z}_{2}$-bundle satisfying $w_{2}(P)+w(\Delta)=0$. Two Spin ${ }^{G}$-bundles have the same image if and only if they are congruent modulo the natural action of $H^{1}\left(X, \mathbb{Z}_{2}\right)$ in $H^{1}\left(X, \underline{\operatorname{Spin}^{G}}\right)$.

Proof: Indeed, the natural morphism $H^{1}\left(X, \underline{S O} \times G / \mathbb{Z}_{2}\right) \longrightarrow H^{2}\left(X, \mathbb{Z}_{2}\right)$ is given by $(P, \Delta) \longmapsto\left(w_{2}(P)+w(\Delta)\right)$.

For instance, we have the following result

Proposition 1.1.2 Let $X$ be a 4-manifold. The group $H^{1}\left(X, \mathbb{Z}_{2}\right)$ acts trivially on the set of (equivalence classes of) $\operatorname{Spin}^{c}(4)$-bundles over $X$. Equivalence classes of $\operatorname{Spin}^{c}(4)$-bundles over $X$ are classified by pairs $(P, \Delta)$ consisting of an $S O(4)$-bundle $P$ and an $S^{1}$-bundle $\Delta$ with $w_{2}(P)+w_{2}(\Delta)=0$.

Proof: Using the identification (see [OT1], [OT3])

$$
\operatorname{Spin}^{c}(4)=\{(a, b) \in U(2) \times U(2) \mid \operatorname{det} a=\operatorname{det} b\},
$$

we get an exact sequence

$$
1 \longrightarrow \operatorname{Spin}^{c}(4) \longrightarrow U(2) \times U(2) \longrightarrow S^{1} \longrightarrow 1 \text {. }
$$

Using this, one can prove that, on 4-manifolds, the data of an (equivalence class of) $\operatorname{Spin}^{c}(4)$-bundles is equivalent to the data of a pair of $U(2)$-bundles 
having isomorphic determinant line bundles. The action of $H^{1}\left(X, \mathbb{Z}_{2}\right)$ is given by tensoring with flat line bundles with structure group $\mathbb{Z}_{2}$. The Chern class of such line bundles is 2-torsion, hence the assertion follows from the classification of unitary vector bundles on 4-manifolds in terms of Chern classes.

The classification of the $S p i n^{G}$-structures in a given $S O$-bundle $P$ is a more delicate problem.

Proposition 1.1.3 Fix a Spin ${ }^{G}$-structure $\sigma: P^{G} \longrightarrow P$ in $P$. Then the set of equivalence classes of Spin $^{G}$-structures in $P$ can be identified with the cohomology set $H^{1}\left(X, \mathbb{G}\left(P^{G}\right)\right)$ of the sheaf of sections in the bundle $\mathbb{G}\left(P^{G}\right)$.

Recall that $\mathbb{G}\left(P^{G}\right)$ can be identified with the bundle $\delta\left(P^{G}\right) \times \overline{\text { Ad }} G$ associated with the $G / \mathbb{Z}_{2}$-bundle $\delta\left(P^{G}\right)$.

Therefore we get the exact sequence of bundles of groups

$$
1 \longrightarrow \mathbb{Z}_{2} \longrightarrow \mathbb{G}\left(P^{G}\right) \longrightarrow \delta\left(P^{G}\right) \times{ }_{\mathrm{Ad}}\left(G / \mathbb{Z}_{2}\right) \longrightarrow 1
$$

The third term coincides with the gauge group of automorphisms of $\delta\left(P^{G}\right)$. The cohomology set $H^{1}\left(X, \delta\left(P^{G}\right) \times{ }_{\overline{A d}}\left(G / \mathbb{Z}_{2}\right)\right)$ of the associated sheaf coincides with the pointed set of (equivalence classes of) $G / \mathbb{Z}_{2}$-bundles over $X$ with distinguished element $\delta\left(P^{G}\right)$. This shows that $H^{1}\left(X, \mathbb{G}\left(P^{G}\right)\right) / H^{1}\left(X, \mathbb{Z}_{2}\right)$ can be identified with the set of $G / \mathbb{Z}_{2}$-bundles $\Delta$ with $w(\Delta)=w\left(\delta\left(P^{G}\right)\right)$. Therefore

Proposition 1.1.4 The map

$$
\left(\sigma: P^{G} \longrightarrow P\right) \longmapsto \delta\left(P^{G}\right)
$$

is a surjection of the set of (equivalence classes of) Spin $^{G}$-structures in $P$ onto the set of $G / \mathbb{Z}_{2}$-bundles $\Delta$ satisfying $w(\Delta)+w_{2}(P)=0$. Two Spin ${ }^{G}$-structures have the same image if and only if they are congruent modulo the natural action of $H^{1}\left(X, \mathbb{Z}_{2}\right)$.

Proposition 1.1.2 and the proposition below show that the classification of $\operatorname{Spin}^{G}$-structures in the $S O$-bundle $P$ is in general different from the classification of $S_{p i n}{ }^{G}$-bundles with associated $S O$-bundle isomorphic to $P$.

Proposition 1.1.5

1. If $G=S^{1}$ then $\operatorname{Spin}^{S^{1}}=\operatorname{Spin}^{c}, \mathbb{G}\left(P^{G}\right)=X \times S^{1}$, hence the set of Spinc-structures in $P$ is a $H^{1}\left(X, \underline{S}^{1}\right)=H^{2}(X, \mathbb{Z})$-torsor if it is non-empty. The $H^{1}\left(X, \mathbb{Z}_{2}\right)$-action in the set of Spin ${ }^{c}$-structures in $P$, factorizes through a $\operatorname{Tors}_{2} H^{2}(X, \mathbb{Z})$-action, which is free and whose orbits coincide with the fibres of the determinant map $\left(\sigma: P^{c} \longrightarrow P\right) \longmapsto \delta\left(P^{c}\right)$. 
2. Suppose that $X$ is a 4-manifold, $P$ is an $S O$-bundle over $X$ and that $G$ is one of the following:

a) $S U(r), r \geq 2, b) U(r), r \geq 2, r$ even. c) $S p(r), r \geq 1$.

Then $H^{1}\left(X, \mathbb{Z}_{2}\right)$ acts trivially in the set of Spin ${ }^{G}$-structures in $P$, hence the classification of $\operatorname{Spin}^{G}$-structures in $P$ reduces to the classification of $G / \mathbb{Z}_{2}$ bundles over $X$.

\section{Proof:}

1. The first assertion follows immediately from Propositions 1.1.3 and 1.1.4.

2. Let $\sigma_{i}: P_{i}^{G} \longrightarrow P, i=0,1$ be two $\operatorname{Spin}^{G}$-structures in $P$. We consider the locally trivial bundle $\operatorname{Iso}_{P}\left(P_{1}^{G}, P_{0}^{G}\right)$ whose fibre in $x \in X$ consists of isomorphism $\rho_{x}:\left(P_{1}^{G}\right)_{x} \longrightarrow\left(P_{0}^{G}\right)_{x}$ of right $\operatorname{Spin}^{G}$-spaces which make the following diagram commutative.

$$
\begin{array}{lll}
\left(P_{1}^{G}\right)_{x} & \stackrel{\rho_{x}}{\longrightarrow} & \left(P_{0}^{G}\right)_{x} \\
\sigma_{0 x} \searrow & & \swarrow \sigma_{1 x} \\
& P_{x} &
\end{array}
$$

$\operatorname{Iso}_{P}\left(P_{1}^{G}, P_{0}^{G}\right)$ is a principal bundle in the sense of Grothendieck with structure group bundle $\mathbb{G}\left(P_{0}^{G}\right)$. The $\operatorname{Spin}^{G}$-structures $\sigma_{i}$ are equivalent if and only if $\operatorname{Iso}_{P}\left(P_{1}^{G}, P_{0}^{G}\right)$ admits a section. Consider first the case $G=S U(r)$ $(r \geq 2)$ or $\operatorname{Sp}(r)(r \geq 1)$. Since $\pi_{i}\left(\left[\operatorname{Iso}_{P}\left(P_{1}^{G}, P_{0}^{G}\right)\right]_{x}\right)=0$ for $i \leq 2$ and $\pi_{3}\left(\left[\operatorname{Iso}_{P}\left(P_{1}^{G}, P_{0}^{G}\right)\right]_{x}\right)$ can be canonically identified with $\mathbb{Z}$, the obstruction $o\left(\sigma_{1}, \sigma_{0}\right)$ to the existence of such a section is an element in $H^{4}(X, \mathbb{Z})$. Assume now that $\sigma_{1}=\lambda \sigma_{0}$ for some $\lambda \in H^{1}\left(X, \mathbb{Z}_{2}\right)$ and let $p: \tilde{X} \longrightarrow X$ the cover associated to $\operatorname{ker} \lambda \subset \pi_{1}(X)$. It is easy to see that one has $o\left(p^{*}\left(\sigma_{1}\right), p^{*}\left(\sigma_{0}\right)\right)=p^{*}\left(o\left(\sigma_{1}, \sigma_{0}\right)\right)$. But, since $p^{*}(\lambda)=0$, we get $p^{*}\left(\sigma_{1}\right)=p^{*}\left(\sigma_{0}\right)$ hence $o\left(p^{*}\left(\sigma_{1}\right), p^{*}\left(\sigma_{0}\right)\right)=0$. Since $p^{*}: H^{4}(X, \mathbb{Z}) \longrightarrow H^{4}(\tilde{X}, \mathbb{Z})$ is injective for a 4-manifold $X$, the assertion follows immediately.

Finally consider $G=U(r)$. When $r \geq 2$ is even, the determinant map $U(r) \longrightarrow S^{1}$ induces a morphism $\operatorname{Spin}^{U(r)} \longrightarrow S^{1}$. If $\sigma_{1}=\lambda \sigma_{0}$, then there is a natural identification $P_{1}^{G} \times{ }_{\operatorname{det}} S^{1}=P_{0}^{G} \times{ }_{\operatorname{det}} S^{1}$, hence, denoting this line bundle by $L$, we get a subbundle $I \operatorname{so}_{P, L}\left(P_{1}^{G}, P_{0}^{G}\right)$ of $\operatorname{Iso}_{P}\left(P_{1}^{G}, P_{0}^{G}\right)$ consisting fibrewise of isomorphisms $\left(P_{1}^{G}\right)_{x} \longrightarrow\left(P_{0}^{G}\right)_{x}$ over $P_{x} \times L_{x}$. Since the standard fibre of $\operatorname{Iso}_{P, L}\left(P_{1}^{G}, P_{0}^{G}\right)$ is $S U(r)$, the same argument as above shows that this bundle admits a section, hence $\sigma_{1}$ and $\sigma_{0}$ are equivalent.

\subsection{2 $\operatorname{Spin}^{G}(4)$-structures on 4-manifolds and spinor bundles}

Let $\mathbb{H}_{ \pm}$be two copies of the quaternionic skewfield, regarded as right quaternionic vector spaces. The canonical left actions of $S p(1)$ in $\mathbb{H}_{ \pm}$define an orthogonal representation of the group

$$
\operatorname{Spin}(4)=S p(1) \times S p(1)=S U(2) \times S U(2)
$$


in $\mathbb{H} \simeq \operatorname{Hom}_{\mathbb{H}}\left(\mathbb{H}_{+}, \mathbb{H}_{-}\right)$, which gives the standard identification

$$
S U(2) \times S U(2) / \mathbb{Z}_{2}=S O(\mathbb{H})=S O(4)
$$

Therefore, the group $\operatorname{Spin}^{G}(4)=S U(2) \times S U(2) \times G / \mathbb{Z}_{2}$ comes with 2 unitary representations

$$
\lambda_{ \pm}: \operatorname{Spin}^{G}(4) \longrightarrow U\left(\mathbb{H}_{ \pm} \otimes_{\mathbb{C}} V\right)
$$

obtained by coupling the natural representation of $G$ in $V$ with the spinorial representations $p_{ \pm}: \operatorname{Spin}(4)=S U(2) \times S U(2) \longrightarrow S U(2)$.

There are well defined adjoint morphisms

$$
\operatorname{ad}_{ \pm}: \operatorname{Spin}^{G}(4) \longrightarrow O(s u(2)), \operatorname{Ad}_{ \pm}: \operatorname{Spin}^{G}(4) \longrightarrow \operatorname{Aut}(S U(2))
$$

induced by the projections $p_{ \pm}$and the corresponding adjoint representations associated with the Lie group $S U(2)$. If $P^{G}$ is a $\operatorname{Spin}^{G}(4)$-bundle, we denote by $\operatorname{ad}_{ \pm}\left(P^{G}\right), \operatorname{Ad}_{ \pm}\left(P^{G}\right)$ the corresponding bundles with fibres $s u(2), S U(2)$ associated with $P^{G}$. The spinor vector bundles associated with a $\operatorname{Spin}^{G}(4)$ bundle $P^{G}$ are defined by

$$
\Sigma^{ \pm}=\Sigma^{ \pm}\left(P^{G}\right):=P^{G} \times_{\lambda_{ \pm}}\left(\mathbb{H}_{ \pm} \otimes_{\mathbb{C}} V\right),
$$

The bundles $\operatorname{ad}_{ \pm}\left(P^{G}\right), \mathfrak{g}\left(P^{G}\right)$ are real subbundles of the endomorphism bundle $\operatorname{End}_{\mathbb{C}}\left(\Sigma^{ \pm}\right)$. The bundle $\mathbb{G}\left(P^{G}\right)$ acts fibrewise unitarily in the bundles $\Sigma^{ \pm}$. On the other hand, the identification $\mathbb{H} \simeq \operatorname{Hom}_{\mathbb{H}}\left(\mathbb{H}_{+}, \mathbb{H}_{-}\right)$defines a real embedding

$$
P^{G} \times_{\pi} \mathbb{H} \longrightarrow \operatorname{Hom}_{\mathbb{G}\left(P^{G}\right)}\left(\Sigma^{+}, \Sigma^{-}\right) \subset \operatorname{Hom}_{\mathbb{C}}\left(\Sigma^{+}, \Sigma^{-}\right)
$$

of the $S O(4)$-vector bundle $P^{G} \times_{\pi} \mathbb{H}$ in the bundle $\operatorname{Hom}_{\mathbb{G}\left(P^{G}\right)}\left(\Sigma^{+}, \Sigma^{-}\right)$of $\mathbb{C}$ linear morphisms $\Sigma^{+} \longrightarrow \Sigma^{-}$which commute with the $\mathbb{G}\left(P^{G}\right)$-action.

The data of a $\operatorname{Spin}^{G}(4)$-structure with principal bundle $P^{G}$ on an oriented Riemannian 4-manifold $X$ is equivalent to the data of an orientation-preserving isomorphism $\Lambda_{X}^{1} \stackrel{\gamma}{\longrightarrow} P^{G} \times_{\pi} \mathbb{H}$, which defines (via the monomorphism in (1)) a Clifford multiplication $\left(\Lambda^{1} \otimes \mathbb{C}\right) \otimes \Sigma^{+} \longrightarrow \Sigma^{-}$commuting with the $\mathbb{G}\left(P^{G}\right)$ actions in $\Sigma^{ \pm}$. Moreover, as in the classical $\operatorname{Spin}^{c}(4)$ case [OT1], [OT3], we also have induced identifications (which multiply the norms by 2 )

$$
\Gamma: \Lambda_{ \pm}^{2} \longrightarrow \operatorname{ad}_{ \pm}\left(P^{G}\right) \text {. }
$$

\subsubsection{Examples}

1. $\operatorname{Spin}^{c}(4)$-structures:

The group $\operatorname{Spin}^{c}(4):=\operatorname{Spin}^{U(1)}(4)$ can be identified with the subgroup

$$
G_{2}:=\{(a, b) \in U(2) \times U(2) \mid \operatorname{det} a=\operatorname{det} b\}
$$


of $U(2) \times U(2)$. Via this identification, the map $\delta: \operatorname{Spin}^{c}(4) \longrightarrow S^{1} \simeq S^{1} / \mathbb{Z}_{2}$ in the exact sequence

$$
1 \longrightarrow \operatorname{Spin}(4) \longrightarrow \operatorname{Spin}^{c}(4) \stackrel{\delta}{\longrightarrow} S^{1} \longrightarrow 1
$$

is given by the formula $\delta(a, b)=\operatorname{det} a=\operatorname{det} b$. The spinor bundles come with identifications

$$
\operatorname{det} \Sigma^{+} \stackrel{\simeq}{\rightrightarrows} \operatorname{det} \Sigma^{-} \stackrel{\simeq}{\rightarrow} P^{c} \times_{\delta} \mathbb{C} .
$$

The $S O(4)$-vector bundle $P^{c} \times_{\pi} \mathbb{H}$ associated with a $\operatorname{Spin}{ }^{c}(4)$-bundle $P^{c}$ can be identified with the bundle $\mathbb{R} S U\left(\Sigma^{+} \Sigma^{-}\right) \subset \operatorname{Hom}\left(\Sigma^{+}, \Sigma^{-}\right)$of real multiples of isometries of determinant 1 .

Using these facts, it easy to see that a $\operatorname{Spin}^{c}(4)$-structure can be recovered from the data of the spinor bundles, the identification between the determinant line bundles and the Clifford map. More precisely

Proposition 1.1.6 The data of a Spinc(4)-structure in the SO(4)-bundle P over $X$ is equivalent to the data of a triple consisting of:

i) A pair of $U(2)$-vector bundles $\Sigma^{ \pm}$.

ii) A unitary isomorphism $\operatorname{det} \Sigma^{+} \stackrel{\iota}{\rightarrow} \operatorname{det} \Sigma^{-}$.

iii) An orientation-preserving linear isometry

$$
\gamma: P \times_{S O(4)} \mathbb{R}^{4} \rightarrow \mathbb{R} S U\left(\Sigma^{+}, \Sigma^{-}\right) .
$$

Proof: Given a triple $\left(\Sigma^{ \pm}, \iota, \gamma\right)$, we define $P^{c}$ to be the manifold over $X$

$$
\begin{aligned}
P^{c}:=\left\{\left[x,\left(e_{1}^{+}, e_{2}^{+}\right),\left(e_{1}^{-}, e_{2}^{-}\right)\right] \mid\right. & x \in X,\left(e_{1}^{ \pm}, e_{2}^{ \pm}\right) \text {an orthonormal basis in } \Sigma_{x}^{ \pm}, \\
& \left.\iota_{*}\left(e_{1}^{+} \wedge e_{2}^{+}\right)=e_{1}^{-} \wedge e_{2}^{-}\right\} .
\end{aligned}
$$

Every triple $\left[x,\left(e_{1}^{+}, e_{2}^{+}\right),\left(e_{1}^{-}, e_{2}^{-}\right)\right] \in P_{x}^{c}$ defines an orthonormal orientationcompatible basis in $\mathbb{R} S U\left(\Sigma_{x}^{+}, \Sigma_{x}^{-}\right)$which is given with respect to the frames $\left(e_{1}^{ \pm}, e_{2}^{ \pm}\right)$by the Pauli matrices. Using the isomorphism $\gamma$, we get a bundle morphism from $P^{c}$ onto the orthonormal oriented frame bundle of $P \times_{S O(4)} \mathbb{R}^{4}$, which can be canonically identified with $P$.

Let $P$ be a principal $S O(4)$-bundle, $P^{c} \stackrel{\mathfrak{c}_{0}}{\longrightarrow} P$ a fixed $\operatorname{Spin}^{c}(4)$-structure in $P, \Sigma^{ \pm}$the associated spinor bundles, and

$$
\gamma_{0}: P \times_{S O(4)} \mathbb{R}^{4} \longrightarrow P^{c} \times_{\pi} \mathbb{H}=\mathbb{R} S U\left(\Sigma^{+}, \Sigma^{-}\right)
$$

the corresponding Clifford map. For every $m \in H^{2}(X, \mathbb{Z})$ let $L_{m}$ be a Hermitian line bundle of Chern class $m$. The fixed identification $\operatorname{det} \Sigma^{+} \stackrel{\sim}{\longrightarrow} \operatorname{det} \Sigma^{-}$induces an identification $\operatorname{det} \Sigma^{+} \otimes L_{m} \stackrel{\sim}{\longrightarrow} \operatorname{det} \Sigma^{-} \otimes L_{m}$, and the map

$$
\gamma_{m}: P \times_{S O(4)} \mathbb{R}^{4} \longrightarrow \mathbb{R} S U\left(\Sigma^{+} \otimes L_{m}, \Sigma^{-} \otimes L_{m}\right), \gamma_{m}(\eta):=\gamma_{0}(\eta) \otimes \operatorname{id}_{L_{m}}
$$

is the Clifford map of a $\operatorname{Spin}^{c}(4)$-structure $\mathfrak{c}_{m}$ in $P$ whose spinor bundles are $\Sigma^{ \pm} \otimes L_{m}$

Using the results in the previous section (see also $[\mathrm{H}],[\mathrm{OT} 1]$, [OT6]) we get 
Proposition 1.1.7

i) An $S O(4)$-bundle $P$ admits a Spinc(4)-structure iff $w_{2}(P)$ admits integral lifts.

ii) The set of isomorphism classes of Spinc(4)-structures in an $S O(4)$ - bundle $P$ is either empty or is an $H^{2}(X, \mathbb{Z})$-torsor. If $\gamma_{0}$ is a fixed $\operatorname{Spin}^{c}(4)$-structure in the $S O(4)$-bundle $P$, then the map $m \longmapsto \mathfrak{c}_{m}$ defines a bijection between $H^{2}(X, \mathbb{Z})$ and the set of (equivalence classes of) Spin ${ }^{c}(4)$-structures in $P$.

iii) $[H H]$ If $(X, g)$ is a compact oriented Riemannian 4-manifold, then $w_{2}\left(P_{g}\right)$ admits integral lifts. In particular any compact oriented Riemannian 4-manifold admits $\operatorname{Spin}^{c}(4)$-structures

2. $\operatorname{Spin}^{h}(4)$-structures:

The quaternionic spin group is defined by $\operatorname{Spin}^{h}:=\operatorname{Spin}^{S p(1)}$. By the classification results 1.1.4., 1.1.5 we get

Proposition 1.1.8 Let $P$ be an SO-bundle over a compact oriented 4-manifold $X$. The map

$$
\left[\sigma: P^{h} \longrightarrow P\right] \longmapsto\left[\delta\left(P^{h}\right)\right]
$$

defines a 1-1 correspondence between the set of isomorphism classes of $\mathrm{Spin}^{h}$ structures in $P$ and the set of isomorphism classes of $P U(2)$-bundles $\bar{P}$ over $X$ with $w_{2}(\bar{P})=w_{2}(P)$. The latter set can be identified ([DK], p.41) with

$$
\left\{p \in \mathbb{Z} \mid p \equiv w_{2}(P)^{2} \bmod 4\right\}
$$

via the Pontrjagin class-map.

In dimension 4, the group $\operatorname{Spin}^{h}(4)$ can be identified with the quotient

$$
S U(2) \times S U(2) \times S U(2) /\{ \pm(\mathrm{id}, \mathrm{id}, \mathrm{id})\},
$$

hence there is an exact sequence

$$
1 \longrightarrow \mathbb{Z}_{2} \longrightarrow S U(2) \times S U(2) \times S U(2) \longrightarrow \operatorname{Spin}^{h}(4) \longrightarrow 1 .
$$

Let $G_{3}$ be the group

$$
G_{3}:=\{(a, b, c) \in U(2) \times U(2) \times U(2) \mid \operatorname{det} a=\operatorname{det} b=\operatorname{det} c\}
$$

We have an exact sequence

$$
1 \longrightarrow S^{1} \longrightarrow G_{3} \longrightarrow \operatorname{Spin}^{h}(4) \longrightarrow 1
$$

extending the exact sequence (2). If $X$ is any manifold, the induced map $H^{1}\left(X, \underline{\operatorname{Spin}^{h}(4)}\right) \longrightarrow H^{2}\left(X, \underline{S^{1}}\right)=H^{3}(X, \mathbb{Z})$ factorizes as

$$
H^{1}\left(X, \underline{\operatorname{Spin}^{h}(4)}\right) \stackrel{\pi}{\longrightarrow} H^{1}(X, \underline{S O(4)}) \stackrel{w_{2}}{\longrightarrow} H^{2}\left(X, \mathbb{Z}_{2}\right) \longrightarrow H^{2}\left(X, S^{1}\right) .
$$


Therefore a $\operatorname{Spin}^{h}(4)$-bundle $P^{h}$ admits an $G_{3}$-reduction iff the second StiefelWhitney class $w_{2}\left(P^{h} \times_{\pi} S O(4)\right)$ of the associated $S O(4)$-bundle admits an integral lift. On the other hand, the data of a $G_{3}$-structure in a $S O(4)$-bundle $P$ is equivalent to the data of a triple consisting of a $\operatorname{Spin}^{c}(4)$-structure $P^{c} \longrightarrow P$ in $P$, a $U(2)$-bundle $E$, and an isomorphism

$$
P^{c} \times_{\delta} \mathbb{C} \stackrel{\sim}{\longrightarrow} \operatorname{det} E .
$$

Therefore ( see [OT5]),

Proposition 1.1.9 Let $P$ be a principal $S O(4)$-bundle whose second StiefelWhitney class $w_{2}(P)$ admits an integral lift. There is a 1-1 correspondence between isomorphism classes of $\operatorname{Spin}^{h}(4)$-structures in $P$ and equivalence classes of triples consisting of a $\operatorname{Spin}^{c}(4)$-structure $P^{c} \longrightarrow P$ in $P$, a $U(2)$-bundle $E$, and an isomorphism $P^{c} \times_{\delta} \mathbb{C} \stackrel{\sim}{\longrightarrow} \operatorname{det} E$.

Two triples are equivalent if, after tensoring the first with an $S^{1}$-bundle, they become isomorphic over $P$.

Let us identify $S U(2) \times S U(2) / \mathbb{Z}_{2}$ with $S O(4)=S O(\mathbb{H})$ as explained above, and denote by

$$
\pi_{i j}: \operatorname{Spin}^{h} \longrightarrow S O(4) \quad 1 \leq i<j \leq 3
$$

the three epimorphisms associated with the three projections of the product $S U(2) \times S U(2) \times S U(2)$ onto $S U(2) \times S U(2)$. Note that $\pi_{12}=\pi$. The spinor bundles $\Sigma^{ \pm}\left(P^{h}\right)$ associated with a principal $\operatorname{Spin}^{h}(4)$-bundle $P^{h}$ are

$$
\Sigma^{+}\left(P^{h}\right)=P^{h} \times_{\pi_{13}} \mathbb{C}^{4}, \quad \Sigma^{-}\left(P^{h}\right)=P^{h} \times_{\pi_{23}} \mathbb{C}^{4}
$$

This shows in particular that the Hermitian 4-bundles $\Sigma^{ \pm}\left(P^{h}\right)$ come with $\underline{\mathrm{a}}$ real structure and compatible trivializations of $\operatorname{det}\left(\Sigma^{ \pm}\left(P^{h}\right)\right)$.

Suppose now that the $\operatorname{Spin}^{h}(4)$-bundle $P^{h}$ admits a $G_{3}$-lifting, consider the associated triple $\left(P^{c}, E, P^{c} \times_{\delta} \mathbb{C} \simeq \operatorname{det} E\right)$, and let $\Sigma^{ \pm}$be the spinor bundles associated with $P^{c}$. The spinor bundles $\Sigma^{ \pm}\left(P^{h}\right)$ of $P^{h}$ and the automorphismbundle $\mathbb{G}\left(P^{h}\right)$ can be be expressed in terms of the $G_{3}$-reduction as follows

$$
\Sigma^{ \pm}\left(P^{h}\right)=\left[\Sigma^{ \pm}\right]^{\vee} \otimes E=\Sigma^{ \pm} \otimes E^{\vee}, \mathbb{G}\left(P^{h}\right)=S U(E) .
$$

Moreover, the associated $P U(2)$-bundle $\delta\left(P^{h}\right)=P^{h} \times{ }_{\delta} P U(2)$ is naturally isomorphic to the $S^{1}$-quotient of the unitary frame bundle $P_{E}$ of $E$.

3. $\operatorname{Spin}^{U(2)}$-structures:

Consider the $U(2)$ spin group

$$
\operatorname{Spin}^{U(2)}:=\operatorname{Spin} \times_{\mathbb{Z}_{2}} U(2),
$$


and let $p: U(2) \longrightarrow P U(2)$ be the canonical projection. The map

$$
p \times \operatorname{det}: U(2) \longrightarrow P U(2) \times S^{1}
$$

induces an isomorphism $U(2) /_{\{ \pm \mathrm{id}\}}=P U(2) \times S^{1}$. Therefore the map $\delta$ : $\operatorname{Spin}^{U(2)} \longrightarrow U(2) /\{ \pm \mathrm{id}\}$ can be written as a pair $(\bar{\delta}$, det) consisting of a $P U(2)-$ and an $S^{1}$-valued morphism. We have exact sequences

$$
\begin{aligned}
& 1 \longrightarrow \operatorname{Spin} \longrightarrow \operatorname{Spin}^{U(2) \stackrel{(\bar{\delta}, \mathrm{det})}{\longrightarrow}} P U(2) \times S^{1} \longrightarrow 1 \\
& 1 \longrightarrow U(2) \longrightarrow \operatorname{Spin}^{U(2)} \stackrel{\pi}{\longrightarrow} S \longrightarrow 1 \\
& 1 \longrightarrow \mathbb{Z}_{2} \longrightarrow \operatorname{Spin}^{U(2) \stackrel{(\pi, \bar{\delta}, \mathrm{det})}{\longrightarrow}} S O \times P U(2) \times S^{1} \longrightarrow 1 \\
& 1 \longrightarrow S U(2) \longrightarrow \operatorname{Spin}^{U(2)} \stackrel{(\pi, \mathrm{det})}{\longrightarrow} S O \times S^{1} \longrightarrow 1 .
\end{aligned}
$$

Let $P^{u} \longrightarrow P$ be a $S p i n^{U(2)}$-structure in a $S O$-bundle $P$ over $X$.

An important role will be played by the subbundles

$$
\mathbb{G}_{0}\left(P^{u}\right):=P^{u} \times{ }_{\operatorname{Ad}_{U(2)}} S U(2), \mathfrak{g}_{0}\left(P^{u}\right):=P^{u} \times{ }_{\operatorname{Ad}_{U(2)}} s u(2)
$$

of $\mathbb{G}\left(P^{u}\right)=P^{u} \times \operatorname{Ad}_{U(2)} U(2), \mathfrak{g}\left(P^{u}\right):=P^{u} \times_{\operatorname{Ad}_{U(2)}} u(2)$ respectively. The group of sections

$$
\mathcal{G}_{0}\left(P^{u}\right):=\Gamma\left(X, \mathbb{G}_{0}\left(P^{u}\right)\right)
$$

in $\mathbb{G}_{0}\left(P^{u}\right)$ can be identified with the group of automorphisms of $P^{u}$ over the $S O \times S^{1}$-bundle $P \times_{X}\left(P^{u} \times_{\operatorname{det}} S^{1}\right)$.

By Propositions 1.1.4, 1.1.5 we get

Proposition 1.1.10 Let $P$ be a principal $S O$-bundle, $\bar{P}$ a $P U(2)$-bundle, and $L$ a Hermitian line bundle over $X$.

i) $P$ admits a Spin $^{U(2)}$-structure $P^{u} \rightarrow P$ with

$$
P^{u} \times_{\bar{\delta}} P U(2) \simeq \bar{P}, \quad P^{u} \times_{\operatorname{det}} \mathbb{C} \simeq L
$$

iff $w_{2}(P)=w_{2}(\bar{P})+\bar{c}_{1}(L)$, where $\bar{c}_{1}(L)$ is the mod 2 reduction of $c_{1}(L)$. ii) If the base $X$ is a compact oriented 4-manifold, then the map

$$
P^{u} \longmapsto\left(\left[P^{u} \times_{\bar{\delta}} P U(2)\right],\left[P^{u} \times_{\operatorname{det}} \mathbb{C}\right]\right)
$$

defines a 1-1 correspondence between the set of isomorphism classes of $\operatorname{Spin}^{U(2)}$ structures in $P$ and the set of pairs of isomorphism classes $([\bar{P}],[L])$, where $\bar{P}$ is a $P U(2)$-bundle and $L$ an $S^{1}$-bundle with $w_{2}(P)=w_{2}(\bar{P})+\bar{c}_{1}(L)$. The latter set can be identified with

$$
\left\{(p, c) \in H^{4}(X, \mathbb{Z}) \times H^{2}(X, \mathbb{Z}) \mid p \equiv\left(w_{2}(P)+\bar{c}\right)^{2} \bmod 4\right\}
$$


The group $\operatorname{Spin}^{U(2)}(4)=S U(2) \times S U(2) \times U(2) /\{ \pm(\mathrm{id}, \mathrm{id}$, id $)\}$ fits in the exact sequence

$$
1 \longrightarrow S^{1} \longrightarrow \tilde{G}_{3} \longrightarrow \operatorname{Spin}^{U(2)}(4) \longrightarrow 1
$$

where

$$
\tilde{G}_{3}:=\{(a, b, c) \in U(2) \times U(2) \times U(2) \mid \operatorname{det} a=\operatorname{det} b\} .
$$

and a $\operatorname{Spin}^{U(2)}(4)$-bundle $P^{u}$ admits a $\tilde{G}_{3}$-reduction iff $w_{2}\left(P^{u} \times_{\pi} S O(4)\right)$ has integral lifts. Therefore, as in Proposition 1.1.9, we get

Proposition 1.1.11 Let $P$ be an $S O(4)$-bundle whose second Stiefel-Whitney class admits integral lifts.

There is a 1-1 correspondence between isomorphism classes of $\operatorname{Spin}^{U(2)}$ structures in $P$ and equivalence classes of pairs consisting of a Spinc ${ }^{(}(4)$-structure $P^{c} \longrightarrow P$ in $P$ and a $U(2)$-bundle $E$. Two pairs are considered equivalent if, after tensoring the first one with a line bundle, they become isomorphic over $P$.

Suppose that the $\operatorname{Spin}^{U(2)}(4)$-bundle $P^{u}$ admits an $\tilde{G}_{3}$-lifting, let $\left(P^{c}, E\right)$ be the pair associated with this reduction, and let $\Sigma^{ \pm}$be the spinor bundles associated with $P^{c}$. Then the associated bundles $\Sigma^{ \pm}\left(P^{u}\right), \bar{\delta}\left(P^{u}\right), \operatorname{det}\left(P^{u}\right)$, $\mathbb{G}\left(P^{u}\right), \mathbb{G}_{0}\left(P^{u}\right)$ can be expressed in terms of the pair $\left(P^{c}, E\right)$ as follows:

$$
\begin{gathered}
\Sigma^{ \pm}\left(P^{u}\right)=\left[\Sigma^{ \pm}\right]^{\vee} \otimes E=\Sigma^{ \pm} \otimes\left(E^{\vee} \otimes\left[\operatorname{det}\left(P^{u}\right)\right]\right), \quad \bar{\delta}\left(P^{u}\right) \simeq P_{E} / S^{1}, \\
\operatorname{det}\left(P^{u}\right) \simeq \operatorname{det}\left(P^{c}\right)^{-1} \otimes(\operatorname{det} E), \quad \mathbb{G}\left(P^{u}\right)=U(E), \mathfrak{g}\left(P^{u}\right)=u(E), \\
\mathbb{G}_{0}\left(P^{u}\right)=S U(E), \mathfrak{g}_{0}\left(P^{u}\right)=s u(E) .
\end{gathered}
$$

\subsection{The G-monopole equations}

\subsubsection{Moment maps for families of complex structures}

Let $(M, g)$ be a Riemannian manifold, and $\mathcal{J} \subset A^{0}\left(s o\left(T_{M}\right)\right)$ a family of complex structures on $M$ with the property that $(M, g, J)$ is a Kähler manifold, for every $J \in \mathcal{J}$. We denote by $\omega_{J}$ the Kähler form of this Kähler manifold. Let $G$ be a compact Lie group acting on $M$ by isometries with are holomorphic with respect to any $J \in \mathcal{J}$. Let $U$ be a fixed subspace of $A^{0}\left(s o\left(T_{M}\right)\right)$ containing the family $\mathcal{J}$, and suppose for simplicity that $U$ is finite dimensional. We define the total $\underline{\text { Kähler }} \underline{\text { form }} \omega_{\mathcal{J}} \in A^{2}\left(U^{\vee}\right)$ by the formula

$$
\left\langle\omega_{\mathcal{J}}, u\right\rangle=g(u(\cdot), \cdot) .
$$


Definition 1.2.1 Suppose that the total Kähler form is closed and G-invariant. A map $\mu: M \longrightarrow \operatorname{Hom}\left(\mathfrak{g}, U^{\vee}\right)$ for will be called a $\mathcal{J}$-moment map for the $G$ action in $X$ if the following two identities hold 1. $\mu(a g)=\left(\operatorname{ad}_{g} \otimes \operatorname{id}_{U^{\vee}}\right)(\mu(a)) \forall a \in M, g \in G$.

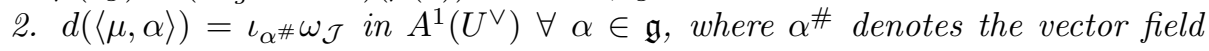
associated with $\alpha$.

In many cases $\mathfrak{g}$ comes with a natural ad-invariant euclidean metric. A map $\mu: M \longrightarrow \mathfrak{g} \otimes U$ will be called also a moment map if its composition with the morphism $\mathfrak{g} \otimes U \longrightarrow \mathfrak{g}^{\vee} \otimes U^{\vee}$ defined by the euclidean structures in $\mathfrak{g}$ and $U$ is a moment map in the above sense. Similarly, the total Kähler form can be regarded (at least in the finite dimensional case) as an element in $\omega_{\mathcal{J}} \in A^{2}(U)$.

Note that if $\mu$ is a moment map with respect to $\mathcal{J}$, then for every $J \in \mathcal{J}$ the map $\mu_{J}:=\langle\mu, J\rangle: M \longrightarrow \mathfrak{g}^{\vee}$ is a moment map for the $G$-action in $X$ with respect to the symplectic structure $\omega_{J}$.

Remark 1.2.2 Suppose that the total Kähler form $\omega_{\mathcal{J}}$ is $G$-invariant and closed. Let $\mu: M \longrightarrow \operatorname{Hom}\left(\mathfrak{g}, U^{\vee}\right)$ be a $\mathcal{J}$-moment map for a free $G$-action and suppose that $\mu$ is a submersion at every point in $\mu^{-1}(0)$. Then $\omega_{\mathcal{J}}$ descend to a closed $U^{\vee}$-valued 2-form on the quotient manifold $\mu^{-1}(0) / G$. In particular, in this case, all the 2-forms $\omega_{J}$ descend to closed 2-formes on this quotient, but they may be degenerate.

\section{Examples:}

\section{Hyperkähler manifolds:}

Let $\left(M, g,\left(J_{1}, J_{2}, J_{3}\right)\right)$ be a hyperkähler manifold [HKLR]. The three complex structures $J_{1}, J_{2}, J_{3}$ span a sub-Lie algebra $U \subset A^{0}\left(\operatorname{so}\left(T_{M}\right)\right)$ naturally isomorphic to $s u(2)$. Suppose for simplicity that $\operatorname{Vol}(M)=1$. The sphere $S(U, \sqrt{2}) \subset U$ of radius $\sqrt{2}$ contains the three complex structures and for any $J \in S(U, \sqrt{2})$ we get a Kähler manifold $(M, g, J)$. Suppose that $G$ acts on $M$ preserving the hyperkähler structure. A hyperkähler moment map $\mu: M \longrightarrow$ $\mathfrak{g} \otimes s u(2)$ in the sense of [HKLR] can be regarded as a moment map with respect to the family $S(U, \sqrt{2})$ in the sense above. If the assumptions in the Remark above are fulfilled, then the forms $\left(\omega_{J}\right)_{J \in S(U, \sqrt{2})}$ descend to symplectic forms on the quotient $\mu^{-1}(0) / G$, which can be endowed with a natural hyperkähler structure in this way [HKLR].

2. Linear hyperkähler spaces: 
Let $G$ be a compact Lie group and $G \subset U(W)$ a unitary representation of $G$. A moment map for the $G$-action on $W$ is given by

$$
\mu_{G}(w)=-\operatorname{Pr}_{\mathfrak{g}}\left(\frac{i}{2}(w \otimes \bar{w})\right)
$$

where $\operatorname{Pr}_{\mathfrak{g}}: u(W) \longrightarrow \mathfrak{g}=\mathfrak{g}^{\vee}$ is the projection $\mathfrak{g} \hookrightarrow u(V)$. Any other moment map can be obtained by adding a constant central element in $\mathfrak{g}$.

In the special case of the standard left action of $S U(2)$ in $\mathbb{C}^{2}$, we denote by $\mu_{0}$ the associated moment map. This is given by

$$
\mu_{0}(x)=-\frac{i}{2}(x \otimes \bar{x})_{0},
$$

where $(x \otimes \bar{x})_{0}$ denotes the trace-free component of the Hermitian endomorphism $x \otimes \bar{x}$.

Consider now the scalar extension $M:=\mathbb{H} \otimes_{\mathbb{C}} W$. Left multiplications by quaternionic units define a $G$-invariant hyperkähler structure in $M$. The corresponding family of complex structures is parametrized by the radius $\sqrt{2}$ sphere $S$ in the space of imaginary quaternions identified with $s u(2)$.

Define the quadratic map $\mu_{0, G}: \mathbb{H} \otimes_{\mathbb{C}} W \longrightarrow s u(2) \otimes \mathfrak{g}$ by

$$
\mu_{0, G}(\Psi)=\operatorname{Pr}_{[s u(2) \otimes \mathfrak{g}]}(\Psi \otimes \bar{\Psi}) .
$$

It acts on tensor monomials by

$$
x \otimes w \stackrel{\mu_{0 G}}{\longmapsto}-4 \mu_{0}(x) \otimes \mu_{G}(w) \in s u(2) \otimes \mathfrak{g} \subset \operatorname{Herm}\left(\mathbb{H} \otimes_{\mathbb{C}} W\right) .
$$

It is easy to see that $-\frac{1}{2} \mu_{0, G}$ is a moment map for the $G$ action in $M$ with respect to the linear hyperkähler structure in $M$ introduced above.

\section{Spaces of spinors:}

Let $P^{G}$ be $\operatorname{Spin}^{G}(4)$-bundle over a compact Riemannian manifold $(X, g)$. The corresponding spinor bundles $\Sigma^{ \pm}\left(P^{G}\right)$ have $\mathbb{H}_{ \pm} \otimes_{\mathbb{C}} V$ as standard fibres. Any section $J \in \Gamma\left(X, S\left(\operatorname{ad}_{ \pm}\left(P^{G}\right), \sqrt{2}\right)\right)$ in the radius $\sqrt{2}$-sphere bundle associated to $a d_{ \pm}\left(P^{G}\right)$ gives a complex (and hence a Kähler) structure in $A^{0}\left(\Sigma^{ \pm}\left(P^{G}\right)\right)$.

Therefore (after suitable Sobolev completions) the space of sections

$$
\Gamma\left(X, S\left(\operatorname{ad}_{ \pm}\left(P^{G}\right), \sqrt{2}\right)\right)
$$

can be regarded as a family of Kähler structures in the space of sections $A^{0}\left(\Sigma^{ \pm}\left(P^{G}\right)\right)$ endowed with the standard $L^{2}$-Euclidean metric. Define a quadratic map $\mu_{0, \mathcal{G}}$ : $A^{0}\left(\Sigma^{ \pm}\left(P^{G}\right)\right) \longrightarrow A^{0}\left(a d_{ \pm}\left(P^{G}\right) \otimes \mathfrak{g}\right)$ by sending an element $\Psi \in A^{0}\left(\Sigma^{ \pm}\left(P^{G}\right)\right)$ to the section in $a d_{ \pm}\left(P^{G}\right) \otimes \mathfrak{g}$ given by the fibrewise projection of $\Psi \otimes \bar{\Psi} \in$ $A^{0}\left(\operatorname{Herm}\left(\Sigma^{ \pm}\left(P^{G}\right)\right)\right)$. 
Then $-\frac{1}{2} \mu_{0, \mathcal{G}}: A^{0}\left(\Sigma^{ \pm}\left(P^{G}\right)\right) \longrightarrow A^{0}\left(\operatorname{ad}_{ \pm}\left(P^{G}\right) \otimes \mathfrak{g}\right) \subset \operatorname{Hom}\left(A^{0}(\mathfrak{g}), A^{0}\left(\operatorname{ad}_{ \pm}\left(P^{G}\right)^{\vee}\right)\right.$ can be regarded as a $\Gamma\left(X, S\left(\operatorname{ad}_{ \pm}\left(P^{G}\right), \sqrt{2}\right)\right)$-moment map for the natural action of the gauge group $\mathcal{G}$.

4. Spaces of connections on a 4-manifold:

Let $(X, g)$ be a compact oriented Riemannian 4-manifold, $G \subset U(r)$ a compact Lie group, and $P$ a principal $G$-bundle over $X$. The space of connections $\mathcal{A}(P)$ is an euclidean affine space modelled on $A^{1}(\operatorname{ad}(P))$, and the gauge group $\mathcal{G}:=\Gamma\left(X, P \times_{A d} G\right)$ acts from the left by $L^{2}$-isometries. The space of almost complex structures in $X$ compatible with the metric and the orientation can be identified with space of sections in the sphere bundle $S\left(\Lambda_{+}^{2}, \sqrt{2}\right)$ under the map which associates to an almost complex structure $J$ the Kähler form $\omega_{J}:=g(\cdot, J(\cdot))[\mathrm{AHS}]$. On the other hand any almost complex structure $J \in \Gamma\left(X, S\left(\Lambda_{+}^{2}, \sqrt{2}\right)\right)$ induces a gauge invariant integrable complex structure in the affine space $\mathcal{A}(P)$ by identifying $A^{1}(\operatorname{ad}(P))$ with $A_{J}^{01}\left(\operatorname{ad}(P)^{\mathbb{C}}\right)$. by

The total Kähler form of this family is the element $\Omega \in A_{\mathcal{A}(P)}^{2}\left(A_{+, X}^{2}\right)$ given

$$
\Omega(\alpha, \beta)=\operatorname{Tr}(\alpha \wedge \beta)^{+},
$$

where $\alpha, \beta \in A^{1}(\operatorname{ad}(P))$.

Consider the map $F^{+}: \mathcal{A}(P) \longrightarrow A^{0}\left(\operatorname{ad}(P) \otimes \Lambda_{X,+}^{2}\right) \subset \operatorname{Hom}\left(A^{0}(\operatorname{ad}(P)),\left(A_{+}^{2}\right)^{\vee}\right)$ given by $A \longmapsto F_{A}^{+}$. It satisfies the equivariance property 1. in Definition 1.2.1. Moreover, for every $A \in \mathcal{A}(P), \alpha \in A^{1}(\operatorname{ad}(P))=T_{A}(\mathcal{A}(P)), \varphi \in A^{0}(\operatorname{ad}(P))=$ $\operatorname{Lie}(\mathcal{G})$ and $\omega \in A_{+}^{2}$ we have (denoting by $\delta$ the exterior derivative on $\mathcal{A}(P)$ )

$$
\left\langle\left(\iota_{\varphi \#} \Omega\right)(\alpha)-\left\langle\delta_{A}\left(F^{+}\right)(\alpha), \varphi\right\rangle, \omega\right\rangle=\left\langle d^{+}[\operatorname{Tr}(\varphi \wedge \alpha)], \omega\right\rangle=\int_{X} \operatorname{Tr}(\varphi \wedge \alpha) \wedge d \omega .
$$

This formula means that the second condition in Definition 1.2.1. holds up to 1-forms on $\mathcal{A}(P)$ with values in the subspace $\operatorname{im}\left[d^{+}: A_{X}^{1} \rightarrow A_{X,+}^{2}\right]$. Let $\bar{\Omega}$ be the image of $\Omega$ in $A_{\mathcal{A}(P)}^{2}\left[A_{X,+}^{2} / \operatorname{im}\left(d^{+}\right)\right]$. Putting

$$
\mathcal{A}_{r e g}^{A S D}=\left\{A \in \mathcal{A}(P) \mid F_{A}^{+}=0, \mathcal{G}_{A}=Z(G), H_{A}^{0}=H_{A}^{2}=0\right\}
$$

we see that $\bar{\Omega}$ descends to a closed $\left[A_{X,+}^{2} / \operatorname{im}\left(d^{+}\right)\right]$-valued 2 -form $[\bar{\Omega}]$ on the moduli space of regular anti-selfdual connections $\mathcal{M}_{\text {reg }}^{A S D}:=\mathcal{A}_{\text {reg }}^{A S D} / \mathcal{G}$. Thus we may consider the map $F^{+}$as a $\Gamma\left(X, S\left(\Lambda_{+}^{2}, \sqrt{2}\right)\right)$-moment map modulo $d^{+}$-exact forms for the action of the gauge group on $\mathcal{A}(P)$.

Note that in the case $G=S U(2)$ taking $L^{2}$-scalar product of $\frac{1}{8 \pi^{2}}[\bar{\Omega}]$ with a harmonic selfdual form $\omega \in \mathbb{H}_{+}^{2}$ defines a de Rham representant of Donaldson's $\mu$-class associated with the Poncaré dual of $[\omega]$. 
The following simple consequence of the above observations can be regarded as the starting point of Seiberg-Witten theory.

Remark 1.2.3 The data of a $\operatorname{Spin}^{G}(4)$-structure in the Riemannian manifold $(X, g)$ gives an isometric isomorphism $\frac{1}{2} \Gamma: \Lambda_{+}^{2} \longrightarrow \operatorname{ad}_{+}\left(P^{G}\right)$. In particular we get an identification between the two familes $\Gamma\left(X, S\left(\operatorname{ad}_{+}\left(P^{G}\right), \sqrt{2}\right)\right)$ and $\Gamma\left(X, S\left(\Lambda_{+}^{2}, \sqrt{2}\right)\right)$ of complex structures in $A^{0}\left(\Sigma^{+}\left(P^{G}\right)\right)$ and $\mathcal{A}\left(\delta\left(P^{G}\right)\right)$ studied before. Consider the action of the gauge group $\mathcal{G}:=\Gamma(X, \mathbb{G})$ on the product $\mathcal{A}\left(\delta\left(P^{G}\right)\right) \times A^{0}\left(\Sigma^{+}\left(P^{G}\right)\right)$ given by

$$
[(A, \Psi), f] \longmapsto(\delta(f)(A), f(\Psi)) .
$$

This action admits a (generalized) moment map modulo $d^{+}$-exact forms (with respect to the family $\Gamma\left(X, S\left(\operatorname{ad}_{+}\left(P^{G}\right), \sqrt{2}\right)\right)$ ) which is given by the formula

$$
(A, \Psi) \longmapsto F_{A}^{+}-\Gamma^{-1}\left(\mu_{0, \mathcal{G}}(\Psi)\right) \text {. }
$$

\subsubsection{Dirac harmonicity and the $G$-monopole equations}

Let $P^{G}$ be a $S p i n^{G}$-bundle. Using the third exact sequence in $(*)$ sect. 1.1, we see that the data of a connection in $P^{G}$ is equivalent to the data of a pair consisting of a connection in $P^{G} \times_{\pi} S O$, and a connection in $\delta\left(P^{G}\right)$. In particular, if $P^{G} \longrightarrow P_{g}$ is a $\operatorname{Spin}^{G}(n)$-structure in the frame bundle of an oriented Riemannian $n$-manifold $X$, then the data of a connection $A$ in $\delta(G)$ is equivalent to the data of a connection $B_{A}$ in $P^{G}$ lifting the LeviCivita connection in $P_{g}$. Suppose now that $n=4$, and denote as usual by $\gamma: \Lambda^{1} \longrightarrow \operatorname{Hom}_{\mathbb{G}}\left(\Sigma^{+}\left(P^{G}\right)^{+}, \Sigma^{-}\left(P^{G}\right)\right)$ the Clifford map of a fixed $\operatorname{Spin}^{G}(4)$ structure $P^{G} \stackrel{\sigma}{\longrightarrow} P_{g}$, and by $\Gamma: \Lambda_{ \pm}^{2} \longrightarrow \operatorname{ad}_{ \pm}\left(P^{G}\right)$ the induced isomorphisms. We define the Dirac operators $\not D_{A}^{ \pm}$associated with $A \in \mathcal{A}\left(\delta\left(P^{G}\right)\right)$ as the composition

$$
A^{0}\left(\Sigma^{ \pm}\left(P^{G}\right)\right) \stackrel{\nabla_{B_{A}}}{\longrightarrow} A^{1}\left(\Sigma^{ \pm}\left(P^{G}\right)\right) \stackrel{\cdot \gamma}{\longrightarrow} A^{0}\left(\Sigma^{\mp}\left(P^{G}\right)\right) .
$$

We put also

$\Sigma\left(P^{G}\right):=\Sigma^{+}\left(P^{G}\right) \oplus \Sigma^{-}\left(P^{G}\right), \not D_{A}:=\not D_{A}^{+} \oplus \not D_{A}^{-}: A^{0}\left(\Sigma\left(P^{G}\right)\right) \longrightarrow A^{0}\left(\Sigma\left(P^{G}\right)\right)$.

Note that $\not D_{A}$ is a self-adjoint first order elliptic operator.

Definition 1.2.4 A pair $(A, \Psi) \in \mathcal{A}\left(P^{G}\right) \times A^{0}\left(\Sigma\left(P^{G}\right)\right)$ will be called (Dirac) harmonic if $\not D_{A} \Psi=0$.

The harmonicity condition is obviously invariant with respect to the gauge group $\mathcal{G}\left(P^{G}\right):=\Gamma\left(X, \mathbb{G}\left(P^{G}\right)\right)$. The monopole equations associated to $\sigma$ couple the two gauge invariant equations we introduced above: the vanishing of the "moment map " (cf. 1.4.1) of the gauge action with respect to the family of complex structures $\Gamma\left(X, S\left(\operatorname{ad}_{+}\left(P^{G}\right), \sqrt{2}\right)\right)$ in the affine space $\mathcal{A}\left(\delta\left(P^{G}\right)\right) \times$ $A^{0}\left(\Sigma^{+}\left(P^{G}\right)\right)$ and the Dirac harmonicity. 
Definition 1.2.5 Let $P^{G} \sigma_{\rightarrow} P$ be a Spin ${ }^{G}(4)$-structure on the compact oriented Riemannian 4-manifold $X$. The associated Seiberg-Witten equations for a pair $(A, \Psi) \in \mathcal{A}\left(\delta\left(P^{G}\right)\right) \times A^{0}\left(\Sigma^{+}\left(P^{G}\right)\right)$ are

$$
\left\{\begin{array}{ccc}
\not D_{A} \Psi & = & 0 \\
\Gamma\left(F_{A}^{+}\right) & = & \mu_{0, \mathcal{G}}(\Psi)
\end{array}\right.
$$

The solutions of these equations modulo the gauge group will be called $G$ monopoles.

The case $G=S^{1}$ corresponds to the classical (abelian) Seiberg-Witten theory. The case $G=S U(2)$ was extensively studied in [OT5], and from a physical point view in $[\mathrm{LM}]$.

Remark 1.2.6 If the Lie algebra $\mathfrak{g}$ of $G$ has non-trivial center $z(\mathfrak{g})$, then the moment map of the gauge action in $A^{0}\left(\Sigma^{+}\left(P^{G}\right)\right)$ is not unique. In this case it is more natural to consider the family of equations

$$
\left\{\begin{array}{ccc}
\not D_{A} \Psi & = & 0 \\
\Gamma\left(F_{A}^{+}\right) & = & \mu_{0, \mathcal{G}}(\Psi)+\beta,
\end{array}\right.
$$

obtained by adding in the second equation a section

$$
\beta \in A^{0}\left(\operatorname{ad}_{+}\left(P^{G}\right) \otimes z(\mathfrak{g})\right) \simeq A_{+}^{2}(X, z(\mathfrak{g})) .
$$

In the case $G=S^{1}$ the equations of this form are called twisted monopole equations [OT6]. If $b_{+}(X)=1$, the invariants defined using moduli spaces of twisted monopoles depend in an essential way on the twisting term $\beta$ ([LL], [OT6]).

The particular case $G=U(2)$ requires a separate discussion, since in this case $\delta(U(2)) \simeq P U(2) \times S^{1}$ and, correspondingly, the bundle $\delta\left(P^{u}\right)$ associated with a $\operatorname{Spin}^{U(2)}(4)$-structure $P^{u} \rightarrow P_{g}$ splits as the product

$$
\delta\left(P^{u}\right)=\bar{\delta}\left(P^{u}\right) \times_{X} \operatorname{det}\left(P^{u}\right)
$$

of a $P U(2)$-bundle with a $U(1)$-bundle. The data of a connection in $P^{u}$ lifting the Levi-Civita connection in $P_{g}$ is equivalent to the data of a pair $A=(\bar{A}, a)$ formed by a connection $\bar{A}$ in $\bar{\delta}\left(P^{u}\right)$ and a connection $a$ in $\operatorname{det}\left(P^{u}\right)$. An alternative approach regards the connection $a \in \mathcal{A}\left(\operatorname{det}\left(P^{u}\right)\right.$ ) as a parameter (not an unknown !) of the equations, and studies the corresponding monopole equations for a pair $(\bar{A}, \Psi) \in \mathcal{A}\left(\bar{\delta}\left(P^{u}\right)\right) \times A^{0}\left(\Sigma^{+}\right)$.

$$
\left\{\begin{array}{llc}
\not D_{\bar{A}, a} \Psi & = & 0 \\
\Gamma\left(F_{\bar{A}}^{+}\right) & = & \mu_{0,0}(\Psi)
\end{array}\right.
$$

Here $\not D_{\bar{A}, a}$ denotes the Dirac operator associated to the connection in $P^{u}$ which lifts the Levi-Civita connection in $P_{g}$, the connection $\bar{A}$ in the $P U(2)$-bundle 
$\bar{\delta}\left(P^{u}\right)$ and the connection $a$ in the $S^{1}$-bundle $\operatorname{det} P^{u}$; the quadratic map $\mu_{0,0}$ sends a spinor $\Psi \in A^{0}\left(\Sigma^{+}\left(P^{u}\right)\right)$ to the projection of the endomorphism $(\Psi \otimes$ $\bar{\Psi}) \in A^{0}\left(\operatorname{Herm}\left(\Sigma^{+}\left(P^{u}\right)\right)\right)$ on $A^{0}\left(\operatorname{ad}_{+}\left(P^{u}\right) \otimes \mathfrak{g}_{0}\left(P^{u}\right)\right)$.

The natural gauge group which lets invariant the equations is the group $\mathcal{G}_{0}\left(P^{u}\right):=\Gamma\left(X, \mathbb{G}_{0}\left(P^{u}\right)\right)$ of automorphisms of the bundle $P^{u}$ over the bundleproduct $P_{g} \times_{X} \operatorname{det}\left(P^{u}\right)$, and $-\mu_{0,0}$ is the $\Gamma\left(X, S\left(\operatorname{ad}_{+}, \sqrt{2}\right)\right)$-moment map for the $\mathcal{G}_{0}\left(P^{u}\right)$-action in the configuration space. There is no ambiguity in choosing the moment map of the $\mathcal{G}_{0}\left(P^{u}\right)$-action, so there is no natural way to perturb these equations besides varying the connection-parameter $a \in \mathcal{A}\left(\operatorname{det}\left(P^{u}\right)\right)$.

Since the connection-component of the unknown is a $P U(2)$-connection, these equations will be called the $P U(2)$-monopole equations, and its solutions modulo the gauge group $\mathcal{G}_{0}\left(P^{u}\right)$ will be called $P U(2)$-monopoles.

Note that if the $\operatorname{Spin}^{U(2)}(4)$-structure $P^{u} \longrightarrow P_{g}$ is associated with the pair $\left(P^{c} \longrightarrow P_{g}, E\right)$ (Proposition 1.1.11), the quadratic map $\mu_{0,0}$ sends a spinor $\Psi \in A^{0}\left(\Sigma^{+}\left(P^{c}\right) \otimes\left[E^{\vee} \otimes \operatorname{det}\left(P^{u}\right)\right]\right)$ to the projection of

$$
(\Psi \otimes \bar{\Psi}) \in A^{0}\left(\operatorname{Herm}\left(\Sigma^{+}\left(P^{c}\right) \otimes\left[E^{\vee} \otimes \operatorname{det}\left(P^{u}\right)\right]\right)\right)
$$

on $A^{0}\left(s u\left(\Sigma^{+}\right) \otimes s u\left(\left[E^{\vee} \otimes \operatorname{det}\left(P^{u}\right)\right]\right)\right.$.

Remark 1.2.7 The data of a $\operatorname{Spin}^{h}(4)$-structure in $X$ is equivalent to the data of Spin ${ }^{U(2)}$-structure $P^{u} \stackrel{\sigma}{\rightarrow} P$ together with a trivialization of the $S^{1}$-bundle $\operatorname{det}\left(P^{u}\right)$. The corresponding $S U(2)$-Seiberg-Witten equations coincide with the $P U(2)$-equations $S W_{\theta}^{\sigma}$ associated with the trivial connection $\theta$ in the trivial bundle $\operatorname{det}\left(P^{u}\right)$.

We shall always regard the $S U(2)$-monopole equations as special $P U(2)$ monopole equations. In particular we shall use the notation $\mu_{0, \mathcal{G}}=\mu_{0,0}$ if $\mathcal{G}$ is the gauge group associated with a $\operatorname{Spin}^{h}(4)$-structure.

Remark 1.2.8 The moduli space of PU(2)-monopoles of the form $(\bar{A}, 0)$ can be identified with a moduli space of anti-selfdual $P U(2)$-connections, modulo the gauge group $\mathcal{G}_{0}$. The natural morphism of $\mathcal{G}_{0}$ into the usual PU(2)-gauge group of bundle automorphisms of $\bar{\delta}\left(P^{u}\right)$ is a local isomorphism but in general it is not surjective (see [LT]). Therefore the space of PU(2)-monopoles of the form $(\bar{A}, 0)$ is a finite cover of the corresponding Donaldson moduli space of $P U(2)-i n s t a n t o n s$.

Remark 1.2.9 Let $G$ be a compact Lie group endowed with a central invlotion $\iota$ and an arbitrary unitary representation $\rho: G \longrightarrow U(V)$ with $\rho(\iota)=-\mathrm{id}_{V}$. One can associate to any $\operatorname{Spin}^{G}(4)$-bundle the spinor bundles $\Sigma^{ \pm}$of standard fibre $\mathbb{H}_{ \pm} \otimes V$. Endow the Lie algebra $\mathfrak{g}$ with an ad-invariant metric. Then one can define $\mu_{0, G}$ using the adjoint of the map $\mathfrak{g} \longrightarrow u(V)$ instead of the orthogonal projection, and the $G$-monopole equations have sense in this more general framework. 


\subsubsection{Reductions}

Let $H \subset G \subset U(V)$ be a closed subgroup of $G$ with $-\mathrm{id}_{V} \in H$. Let $P^{G} \rightarrow P$ be a $\operatorname{Spin}^{G}$-structure in the $S O$-bundle bundle $P$.

Definition 1.2.10 A Spin ${ }^{H}$-reduction of $\sigma$ is a subbundle $P^{H}$ of $P^{G}$ with structure group $\operatorname{Spin}^{H} \subset \operatorname{Spin}^{G}$.

Note that such a reduction $P^{H} \hookrightarrow P^{G}$ defines a reduction $\delta\left(P^{H}\right) \hookrightarrow \delta\left(P^{G}\right)$ of the structure group of the bundle $\delta\left(P^{G}\right)$ from $G / \mathbb{Z}_{2}$ to $H / \mathbb{Z}_{2}$, hence it defines in particular an injective linear morphism $\mathcal{A}\left(\delta\left(P^{H}\right)\right) \hookrightarrow \mathcal{A}\left(\delta\left(P^{G}\right)\right)$ between the associated affine spaces of connections.

Let now $V_{0}$ be an $H$-invariant subspace of $V$.

Consider a $\operatorname{Spin}^{G}(4)$-structure $P^{G} \rightarrow P$ in the $S O(4)$-bundle $P$, and a $\operatorname{Spin}^{H}(4)$-reduction $P^{H} \stackrel{\rho}{\hookrightarrow} P^{G}$ of $\sigma$. Let $\Sigma^{ \pm}\left(P^{H}, V_{0}\right)$ be the spinor bundles associated with $P^{H}$ and the $\operatorname{Spin}^{H}(4)$-representation in $\mathbb{H}^{ \pm} \otimes_{\mathbb{C}} V_{0}$.

The inclusion $V_{0} \subset V$ induces bundle inclusions of the associated spinor bundles $\Sigma^{ \pm}\left(P^{H}, V_{0}\right) \hookrightarrow \Sigma^{ \pm}\left(P^{G}\right)$. Suppose now that $P_{g}$ is the frame-bundle of a compact oriented Riemannian 4-manifold, choose $A \in \mathcal{A}\left(\delta\left(P^{H}\right)\right) \subset \mathcal{A}\left(\delta\left(P^{G}\right)\right)$, and let be $B_{A} \in \mathcal{A}\left(\delta\left(P^{G}\right)\right)$ be the induced connection. Then the spinor bundles $\Sigma^{ \pm}\left(P^{H}, V_{0}\right)$ become $B_{A}$-parallel subbundles of $\Sigma^{ \pm}\left(P^{G}\right)$, and the Dirac operator

$$
\not D_{A}: \Sigma\left(P^{G}\right) \longrightarrow \Sigma\left(P^{G}\right)
$$

maps $\Sigma\left(P^{H}, V_{0}\right)$ into itself. Therefore the set of Dirac-harmonic pairs associated with $\left(\sigma \circ \rho, V_{0}\right)$ can be identified with a subset of the set of Dirac-harmonic pairs associated with $(\sigma, V)$.

The group $G$ acts on the set

$$
\left\{\left(H, V_{0}\right) \mid H \subset G \text { closed subgroup, } V_{0} \subset V \text { is } H \text { - invariant }\right\} .
$$

of subpairs of $(G, V)$ by $\left[g,\left(H, V_{0}\right)\right] \longmapsto\left(A d_{g}(H), g\left(V_{0}\right)\right)$. Moreover, for any $\operatorname{Spin}^{H}(4)$-reduction $P^{H} \hookrightarrow P^{G}$ of $\sigma$ and any element $g \in G$ we get a reduction $P^{\operatorname{Ad}_{g}(H)} \hookrightarrow P^{G}$ of $\sigma$ and subbundles $\Sigma^{ \pm}\left(P^{\operatorname{Ad}_{g}(H)}, g\left(V_{0}\right)\right)$ of the spinor bundles $\Sigma^{ \pm}\left(P^{G}\right)$.

Definition 1.2.11 A subpair $\left(H, V_{0}\right)$ of $(G, V)$ with $-\mathrm{id}_{V} \in H$ will be called admissible and $\left.\mu_{G}\right|_{V_{0}}$ takes values in $\mathfrak{h}$ or, equivalently, if $\langle i k(v), v\rangle=0$ for all $k \in \mathfrak{h}^{\perp \mathfrak{g}}$ and $v \in V_{0}$.

Therefore, if $\left(H, V_{0}\right)$ is admissible, then $\left.\mu_{G}\right|_{V_{0}}$ can be identified with the moment map $\mu_{H}$ associated with the $H$-action in $V_{0}$ (with respect to the metric in hinduced from $\mathfrak{g}-$ see Remark 1.2.9). If generally $E$ is a system of equations on a configuration space $\mathcal{A}$ we denote by $\mathcal{A}^{E}$ the space of solutions of this system, enowed with the induced topology. 
Proposition 1.2.12 Let $\left(H, V_{0}\right)$ be an admissible subpair of $(G, V)$. A Spin $^{H}(4)$-reduction $P^{H} \stackrel{\rho}{\rightarrow} P^{G}$ of the $\operatorname{Spin}{ }^{G}(4)$-structure $P^{G} \stackrel{\sigma}{\longrightarrow} P_{g}$ induces an inclusion

$$
\left[\mathcal{A}\left(\delta\left(P^{H}\right)\right) \times A^{0}\left(\Sigma^{+}\left(P^{H}\right)\right)\right]^{S W^{\sigma o \rho}} \subset\left[\mathcal{A}\left(\delta\left(P^{G}\right)\right) \times A^{0}\left(\Sigma^{+}\left(P^{G}\right)\right)\right]^{S W^{\sigma}}
$$

which is equvariant with respect to the actions of the two gauge groups.

Definition 1.2.13 Let $\left(H, V_{0}\right)$ be an admissible subpair. A solution $(A, \Psi) \in$ $\left[\mathcal{A}\left(\delta\left(P^{G}\right)\right) \times A^{0}\left(\Sigma^{+}\left(P^{G}\right)\right)\right]^{S W^{\sigma}}$ will be called reducible of type $\left(H, V_{0}\right)$, if it belongs to the image of such an inclusion, for a suitable reduction $P^{H} \stackrel{\rho}{\rightarrow} P^{G}$.

If $\left(H, V_{0}\right)$ is admissible, $H \subset H^{\prime}$ and $V_{0}$ is $H^{\prime}$-invariant, then $\left(H^{\prime}, V_{0}\right)$ is also admissible. An admissible pair $\left(H, V_{0}\right)$ will be called minimal if $H$ is minimal in the set of closed subgroups $H^{\prime} \subset G$ such that $\left(H^{\prime}, V_{0}\right)$ is an admissible subpair of $(G, V)$. The sets of (minimal) admissible pairs is invariant under the natural $G$-action. We list the conjugacy classes of proper minimal admissible subpairs in the cases $\left(S U(2), \mathbb{C}^{\oplus 2}\right)=(S p(1), \mathbb{H}),\left(U(2), \mathbb{C}^{\oplus 2}\right),\left(S p(2), \mathbb{H}^{\oplus 2}\right)$. Fix first the maximal tori

$$
\begin{gathered}
T_{S U(2)}:=\left\{\left(\begin{array}{cc}
z & 0 \\
0 & z^{-1}
\end{array}\right) \mid z \in S^{1}\right\}, T_{U(2)}:=\left\{\left(\begin{array}{ll}
u & 0 \\
0 & v
\end{array}\right) \mid u, v \in S^{1}\right\} \\
T_{S p(2)}:=\left\{\left(\begin{array}{ll}
u & 0 \\
0 & v
\end{array}\right) \mid u, v \in S^{1}\right\}
\end{gathered}
$$

On the right we list the minimal admissible subpairs of the pair on the left:

$$
\begin{aligned}
& \left(S U(2), \mathbb{C}^{\oplus 2}\right): \quad(\{ \pm 1\} \quad, \quad\{0\}) \\
& \left(T_{S U(2)} \quad, \quad \mathbb{C} \oplus\{0\}\right) \\
& \left(U(2), \mathbb{C}^{\oplus 2}\right): \quad(\{ \pm 1\} \quad, \quad\{0\}) \\
& \left(\left\{\left(\begin{array}{cc}
\zeta & 0 \\
0 & \pm 1
\end{array}\right) \mid \zeta \in S^{1}\right\} \quad, \quad \mathbb{C} \times\{0\}\right) \\
& \left(S p(2), \mathbb{H}^{\oplus 2}\right): \quad(\{ \pm 1\} \quad, \quad\{0\})
\end{aligned}
$$

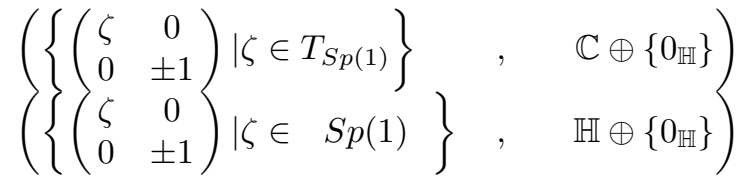


Remark 1.2.14 Fix a maximal torus $T$ of $G$ with Lie algebra $\mathfrak{t}$, and let $\mathfrak{W} \subset \mathfrak{t}^{\vee}$ be the weights of the induced $T$-action in $V$. Let $V=\bigoplus_{\mathfrak{W}} V_{\alpha}$ be the corre$\alpha \in \mathfrak{W}$ sponding decomposition of $V$ in weight spaces. If $\left(T, V^{\prime}\right)$ is a subpair of $(G, V)$, then $V^{\prime}$ must be a sum of weight subspaces, i.e. there exist $\mathfrak{W}^{\prime} \subset \mathfrak{W}$ such that $V^{\prime}=\bigoplus V_{\alpha}^{\prime}$, with $0 \neq V_{\alpha}^{\prime} \subset V_{\alpha}$. When $G$ is one of the classical groups $S U(n)$, $\alpha \in \mathfrak{W}^{\prime}$

$U(n), S p(n)$ and $V$ the corresponding canonical $G$-module, it follows easily that $\left(T, V^{\prime}\right)$ is admissible iff $\left|\mathfrak{W}^{\prime}\right|=1$. Notice that there is a natural action of the Weil group $N(T) / T$ in the set of abelian subpairs of the form $\left(T, V^{\prime}\right)$.

The case of the $P U(2)$-monopole equations needs a separate discussion: Fix a $\operatorname{Spin}^{U(2)}(4)$-structure $\sigma: P^{u} \longrightarrow P_{g}$ in $P_{g}$ and a connection $a$ in the line bundle $\operatorname{det}\left(P^{u}\right)$. In this case the admissible pairs are by definition equivalent to one of

$$
(H,\{0\}), \quad H \subset U(2) \text { with }-\operatorname{id}_{V} \in H ; \quad\left(T_{U(2)}, \mathbb{C} \oplus\{0\}\right)
$$

An abelian reduction $P^{T_{U(2)}} \stackrel{\rho}{\hookrightarrow} P^{u}$ of $\sigma$ gives rise to a pair of $S p i n^{c_{-}}$ structures $\left(\mathfrak{c}_{1}: P_{1}^{c} \longrightarrow P_{g}, \mathfrak{c}_{2}: P_{2}^{c} \longrightarrow P_{g}\right)$ whose determinant line bundles come with an isomorphism $\operatorname{det}\left(P_{1}^{c}\right) \otimes \operatorname{det}\left(P_{2}^{c}\right)=\left[\operatorname{det}\left(P^{u}\right)\right]^{2}$. Moreover, the $P U(2)$-bundle $\bar{\delta}\left(P^{u}\right)$ comes with an $S^{1}$-reduction $\bar{\delta}\left(P^{u}\right)=P^{S^{1}} \times_{\alpha} P U(2)$ where $\left[P^{S^{1}}\right]^{2}=\operatorname{det}\left(P_{1}^{c}\right) \otimes \operatorname{det}\left(P_{2}^{c}\right)^{-1}$ and $\alpha: S^{1} \longrightarrow P U(2)$ is the standard embedding $\zeta \longmapsto\left[\left(\begin{array}{ll}\zeta & 0 \\ 0 & 1\end{array}\right)\right]$. Since we have fixed the connection $a$ in $\operatorname{det}\left(P^{u}\right)$, the data of a connection $\bar{A} \in \mathcal{A}\left(\bar{\delta}\left(P^{u}\right)\right)$ which reduces to $P^{T_{U(2)}}$ via $\rho$ is equivalent to the data of a connection $a_{1} \in \mathcal{A}\left(\operatorname{det}\left(P_{1}^{c}\right)\right)$.

Moreover, we have a natural parallel inclusion $\Sigma^{ \pm}\left(P_{1}^{c}\right) \subset \Sigma^{ \pm}\left(P^{u}\right)$. Consider the following twisted abelian monopole equations [OT6] for a pair $\left(A_{1}, \Psi_{1}\right) \in$ $\mathcal{A}\left(\operatorname{det}\left(P_{1}^{c}\right)\right) \times A^{0}\left(\Sigma^{ \pm}\left(P_{1}^{c}\right)\right)$

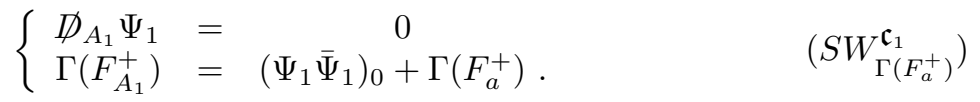

Taking in Remark 1.2.6 as twisting term the form $\beta=\Gamma\left(F_{a}^{+}\right)$, we get

Proposition 1.2.15 A $\operatorname{Spin}^{T_{U(2)}}$-reduction $P^{T_{U(2)}} \stackrel{\rho}{\hookrightarrow} P^{u}$ of the $\operatorname{Spin}^{U(2)}(4)$ structure $P^{u} \rightarrow P_{g}$ induces an inclusion

$$
\left[\mathcal{A}\left(\operatorname{det}\left(P_{1}^{c}\right)\right) \times A^{0}\left(\Sigma^{+}\left(P_{1}^{c}\right)\right)\right]^{S W_{\left(F_{a}^{+}\right)}^{\mathfrak{c}_{1}}} \subset\left[\mathcal{A}\left(\bar{\delta}\left(P^{u}\right)\right) \times A^{0}\left(\Sigma^{+}\left(P^{u}\right)\right)\right]^{S W_{a}^{\sigma}}
$$

which is equivariant with respect to the actions of the two gauge groups.

The fact that the Donaldson $(P U(2)-) S U(2)$-moduli space is contained in the space of $(P U(2)-) S U(2)$-monopoles, and that (twisted) abelian monopoles arise as abelian reductions in the space of $(P U(2)) S U(2)$-monopoles suggests 
that these moduli spaces can be used to prove the equivalence between the two theories [OT5].

This idea can be applied to get information about the Donaldson invariants associated with larger symmetry groups $G$ by relating these invariants to Seiberg-Witten type invariants associated with smaller symmetry groups. In order to do this, one has first to study invariants associated to the moduli spaces of reducible solutions of all possible types in a suitable moduli space of $G$-monopoles.

\subsubsection{Moduli spaces of $G$-monopoles}

Let $\mathcal{A}$ be the configuration space of one of the monopole equations $S W$ introduced in sect. 1.2.2.: For the equations $S W_{\beta}^{\sigma}$ associated with a $\operatorname{Spin}^{G}(4)$ structure $\sigma: P^{G} \longrightarrow P_{g}$ in $(X, g)$ and a section $\beta \in A^{0}\left(\operatorname{ad}_{+}\left(P^{G}\right) \otimes z(\mathfrak{g})\right)$, the space $\mathcal{A}$ coincides with $\mathcal{A}\left(\delta\left(P^{G}\right)\right) \times A^{0}\left(\Sigma^{+}\left(P^{G}\right)\right)$; in the case of $P U(2)$ monopole equations $S W_{a}^{\sigma}$ associated to a $\operatorname{Spin}^{U(2)}(4)$-structure $\sigma: P^{u} \longrightarrow P_{g}$ and an abelian connection $a \in \mathcal{A}\left(\operatorname{det}\left(P^{u}\right)\right)$ the configuration space is $\mathcal{A}\left(\bar{\delta}\left(P^{u}\right)\right) \otimes$ $A^{0}\left(\Sigma^{+}\left(P^{u}\right)\right)$. In this section, we denote by $\mathcal{G}$ the gauge group corresponding to the monopole equation $S W$, i.e. $\mathcal{G}=\mathcal{G}\left(P^{G}\right)$ if $S W=S W_{\beta}^{\sigma}$ and $\mathcal{G}=\mathcal{G}_{0}\left(P^{u}\right)$ in the $P U(2)$-case $S W=S W_{a}^{\sigma}$. The Lie algebra $\operatorname{Lie}(\mathcal{G})$ of $\mathcal{G}$ is $\Gamma\left(X, \mathfrak{g}\left(P^{G}\right)\right)$ in the first case and $\Gamma\left(X, \mathfrak{g}_{0}\left(P^{u}\right)\right)$ in the second.

The corresponding moduli space of $G$-monopoles is defined as a topological space by

$$
\mathcal{M}:=\mathcal{A}^{S W} / \mathcal{G}
$$

There is a standard way of describing the local structure of $\mathcal{M}$, which was extensively described in the cases $G=S^{1}, G=U(2)$ in [OT1] and in the case $G=S U(2)$ (which is similar to the $P U(2)$-case) in [OT5] (see [DK], [K], [LT], $[\mathrm{M}]$ for the instanton case and for the classical case of holomorphic bundles). We explain briefly the general strategy:

Let $p=(A, \Psi) \in \mathcal{A}^{S W}$. The infinitesimal action of $\operatorname{Lie}(\mathcal{G})$ and the differential of $S W$ in $p$ define a "elliptic deformation complex"

$$
0 \longrightarrow C_{p}^{0} \stackrel{D_{p}^{0}}{\longrightarrow} C_{p}^{1} \stackrel{D_{p}^{1}}{\longrightarrow} C_{p}^{2} \longrightarrow 0
$$

where:

$C_{p}^{0}=\operatorname{Lie}(\mathcal{G})=\Gamma\left(X, \mathfrak{g}\left(P^{G}\right)\right)\left(\right.$ or $\Gamma\left(X, \mathfrak{g}_{0}\left(P^{u}\right)\right)$ in the $P U(2)$-case $)$,

$\mathcal{C}_{p}^{1}=T_{p}(\mathcal{A})=A^{1}\left(\mathfrak{g}\left(P^{G}\right)\right) \oplus A^{0}\left(\Sigma^{+}\left(P^{G}\right)\right)\left(\right.$ or $A^{1}\left(\mathfrak{g}_{0}\left(P^{u}\right)\right) \oplus A^{0}\left(\Sigma^{+}\left(P^{u}\right)\right)$ in the $P U(2)$-case),

$C_{p}^{2}=A^{0}\left(\operatorname{ad}_{+}\left(P^{G}\right) \otimes \mathfrak{g}\left(P^{G}\right)\right) \oplus A^{0}\left(\Sigma^{-}\left(P^{G}\right)\right)\left(\operatorname{or} A^{0}\left(\operatorname{ad}_{+}\left(P^{u}\right) \otimes \mathfrak{g}_{0}\left(P^{u}\right)\right) \oplus A^{0}\left(\Sigma^{-}\left(P^{u}\right)\right)\right.$ in the $P U(2)$-case), 
$D_{p}^{0}(f):=f_{p}^{\#}=\left(-d_{A} f, f \Psi\right)$,

$D_{p}^{1}(\alpha, \psi):=d_{p} S W(\alpha, \psi)=\left(\Gamma\left(d_{A}^{+} \alpha\right)-m(\psi, \Psi)-m(\Psi, \psi), \gamma(\alpha) \Psi+\not D_{A}(\psi)\right)$. Here $m$ is the sesquilinear map associated with the quadratic map $\mu_{0, \mathcal{G}}$ (or $\mu_{0,0}$ in the $P U(2)$-case).

The index $\chi$ of this elliptic complex is called the expected dimension of the moduli space and can be easily computed by Atiyah-Singer Index-Theorem [LMi] in terms of characteristic classes of $X$ and vector bundles associated with $P^{G}$.

We give the result in the case of the $P U(2)$-monopole equations:

$$
\chi\left(S W_{a}^{\sigma}\right)=\frac{1}{2}\left(-3 p_{1}\left(\bar{\delta}\left(P^{u}\right)\right)+c_{1}\left(\operatorname{det}\left(P^{u}\right)\right)^{2}\right)-\frac{1}{2}(3 e(X)+4 \sigma(X))
$$

The same methods as in [OT5] give:

\section{Proposition 1.2.16}

1. The stabilizer $\mathcal{G}_{p}$ of $p$ is a finite dimensional Lie group isomorphic to a subgroup of $G$ which acts in a natural way in the harmonic spaces $\mathbb{H}^{i}\left(\mathcal{C}_{p}\right), i=$ $0,1,2$.

2. There exists a neighbourhood $V_{p}$ of $P$ in $\mathcal{M}$, a $\mathcal{G}_{p}$-invariant neighbourhood $U_{p}$ of 0 in $\mathbb{H}^{1}\left(\mathcal{C}_{p}\right)$, a $\mathcal{G}_{p}$-equivariant real analytic map $K_{p}: U_{p} \longrightarrow \mathbb{H}^{2}\left(\mathcal{C}_{p}\right)$ with $K_{p}(0)=0, d K_{p}(0)=0$ and a homeomorphism:

$$
V_{p} \simeq Z\left(K_{p}\right) / \mathcal{G}_{p} .
$$

The homeomorphisms in the proposition above define a structure of a smooth manifold of dimension $\chi$ in the open set

$$
\mathcal{M}_{\text {reg }}=\left\{[p] \in \mathcal{M} \mid \mathcal{G}_{p}=\{1\}, H^{2}\left(\mathcal{C}_{p}\right)=0\right\}
$$

of regular points, and a structure of a real analytic orbifold in the open set of points with finite stabilizers.

Note that the stabilizer of a solution of the form $(A, 0)$ contains always $\{ \pm$ id $\}$, hence $\mathcal{M}$ has at least $\mathbb{Z}_{2}$-orbifold singularities in the Donaldson points (see Remark 1.2.8).

As in the instanton case, the moduli space $\mathcal{M}$ is in general non-compact. The construction of an Uhlenbeck-type compactification is treated in [T1], [T2].

\section{$2 \quad P U(2)-$ Monopoles and stable oriented pairs}

In this section we show that the moduli spaces of $P U(2)$-monopoles on a compact Kähler surface have a natural complex geometric description in terms of stable oriented pairs. We explain first briefly, following [OT5], the concept of oriented pair and we indicate how moduli space of simple oriented pairs are constructed. 
Next we restrict ourselves to the rank 2-case and we introduce the concept of stable oriented pair; the stability property we need [OT5] does not depend on a parameter and is an open property. An algebraic geometric approach can be found in [OST].

In section 2.2 we give a complex geometric description of the moduli spaces of irreducible $P U(2)$-monopoles on a Kähler surface in terms of moduli spaces of stable oriented pairs. This description is used to give an explicit description of a moduli space of $P U(2)$-monopoles on $\mathbb{P}^{2}$.

\subsection{Simple, strongly simple and stable oriented pairs}

Let $(X, g)$ be a compact Kähler manifold of dimension $n, E$ a differentiable vector bundle of rank $r$ on $X$, and $\mathcal{L}=\left(L, \bar{\partial}_{\mathcal{L}}\right)$ a fixed holomorphic structure in the determinant line bundle $L:=\operatorname{det} E$. We recall (see [OT5]) the following fundamental definition:

Definition 2.1.1 An oriented pair of type $(E, \mathcal{L})$ is a pair $(\mathcal{E}, \varphi)$, where $\mathcal{E}$ is a holomorphic structure in $E$ such that $\operatorname{det} \mathcal{E}=\mathcal{L}$, and $\varphi \in H^{0}(\mathcal{E})$. Two oriented pairs $\left(\mathcal{E}_{1}, \varphi_{1}\right),\left(\mathcal{E}_{2}, \varphi_{2}\right)$ of type $(E, \mathcal{L})$ are called isomorphic if they are congruent modulo the natural action of the group $\Gamma(X, S L(E))$ of differentiable automorphism of $E$ of determinant 1.

Therefore we fix the underlying $\mathcal{C}^{\infty}$-bundle and the holomorphic determinant line bundle (not only its isomorphism type !) of the holomorphic bundles we consider.

An oriented pair $p=(\mathcal{E}, \varphi)$ is called simple if its stabilizer $\Gamma(X, S L(E))_{p}$ is contained in the center $\mathbb{Z}_{r}$ id $\mathrm{d}_{E}$ of $\Gamma(X, S \overline{L(E))}$, and is called strongly simple if its stabilizer is trivial.

The first property has an equivalent infinitesimal formulation: the pair $(\mathcal{E}, \varphi)$ is simple if and only if any trace-free holomorphic endomorphism of $\mathcal{E}$ with $f(\varphi)=0$ vanishes.

In [OT5] it was shown that

Proposition 2.1.2 There exists a (possibly non-Hausdorff) complex analytic orbifold $\mathcal{M}^{s}(E, \mathcal{L})$ parameterizing isomorphism classes of simple oriented pairs of type $(E, \mathcal{L})$. The open subset $\mathcal{M}^{s s}(E, \mathcal{L}) \subset \mathcal{M}^{s}(E, \mathcal{L})$ consisting of strongly simple pairs is a complex analytic space, and the points in $\mathcal{M}^{s}(E, \mathcal{L}) \backslash \mathcal{M}^{s s}(E, \mathcal{L})$ have neighbourhoods modeled on $\mathbb{Z} / r$-quotients.

If $\mathcal{E}$ is holomorphic bundle we denote by $\mathcal{S}(\mathcal{E})$ the set of reflexive subsheaves $\mathcal{F} \subset \mathcal{E}$ with $0<\operatorname{rk}(\mathcal{F})<\operatorname{rk}(\mathcal{E})$. Once we have fixed a section $\varphi \in H^{0}(\mathcal{E})$, we put

$$
\mathcal{S}_{\varphi}(\mathcal{E}):=\left\{\mathcal{F} \in \mathcal{S}(\mathcal{E}) \mid \varphi \in H^{0}(X, \mathcal{F})\right\}
$$

We recall (see $[\mathrm{B}]$ ) that $\mathcal{E}$ is called $\varphi$-stable if 


$$
\max \left(\mu_{g}(\mathcal{E}), \sup _{\mathcal{F}^{\prime} \in \mathcal{S}(\mathcal{E})} \mu_{g}\left(\mathcal{F}^{\prime}\right)\right)<\inf _{\mathcal{F} \in \mathcal{S}_{\varphi}(\mathcal{E})} \mu_{g}(\mathcal{E} / \mathcal{F})
$$

where for a nontrivial torsion free coherent sheaf $\mathcal{F}, \mu_{g}(\mathcal{F})$ denotes its slope with respect to the Kähler metric $g$. If the real number $\lambda$ belongs to the interval $\left(\max \left(\mu_{g}(\mathcal{E}), \sup _{\mathcal{F}^{\prime} \in \mathcal{S}(\mathcal{E})} \mu_{g}\left(\mathcal{F}^{\prime}\right)\right), \inf _{\mathcal{F} \in \mathcal{S}_{\varphi}(\mathcal{E})} \mu_{g}(\mathcal{E} / \mathcal{F})\right)$, the pair $(\mathcal{E}, \varphi)$ is called $\lambda$-stable.

If $\mathcal{M}$ is a holomorphic line bundle and $\varphi \in H^{0}(\mathcal{M}) \backslash\{0\}$, then $(\mathcal{M}, \varphi)$ is $\lambda$-stable iff $\mu_{g}(\mathcal{M})<\lambda$.

The correct definition of the stability property for oriented pairs of arbitrary rank is a delicate point [OST]. The definition must agree in the algebraicprojective case with the corresponding GIT-stability condition. On the other hand, in the case $r=2$ the definition simplifies considerably and this case is completely sufficient for our purposes. Therefore from now on we assume $r=2$, and we recall from [OT5] the following

\section{Definition 2.1.3}

An oriented pair $(\mathcal{E}, \varphi)$ of type $(E, \mathcal{L})$ is called stable if one of the following conditions holds:

I. $\mathcal{E}$ is $\varphi$-stable,

II. $\varphi \neq 0$ and $\mathcal{E}$ splits in direct sum of line bundles $\mathcal{E}=\mathcal{E}_{1} \oplus \mathcal{E}_{2}$, such that $\varphi \in H^{0}\left(\mathcal{E}_{1}\right)$ and the pair $\left(\mathcal{E}_{1}, \varphi\right)$ is $\mu_{g}(E)$-stable.

$A$ holomorphic pair $(\mathcal{E}, \varphi)$ of type $(E, \mathcal{L})$ is called polystable if it is stable, or $\varphi=0$ and $\mathcal{E}$ is a polystable bundle.

Remark 2.1.4 An oriented pair $(\mathcal{E}, \varphi)$ of type $(E, \mathcal{L})$ with $\varphi \neq 0$ is stable iff $\mu_{g}\left(\mathcal{O}_{X}\left(D_{\varphi}\right)\right)<\mu_{g}(E)$, where $D_{\varphi}$ is the divisorial component of the vanishing locus $Z(\varphi)$. An oriented pair of the form $(\mathcal{E}, 0)$ is stable iff the holomorphic bundle $\mathcal{E}$ is stable.

\subsection{The projective vortex equation and stability of ori- ented pairs}

The stability property for holomorphic bundles has a well known differential geometric characterization: an holomorphic bundle is stable if and only if it is simple and admits a Hermite-Einstein metric (see for instance [DK], [LT]). Similarly, an holomorphic pair $(\mathcal{E}, \varphi)$ is $\lambda$-stable if and only it is simple and $\mathcal{E}$ admits a Hermitian metric satisfying the vortex equation associated with the constant $t=\frac{4 \pi \lambda}{\operatorname{Vol}_{g}(X)}[\mathrm{B}]$. All these important results are infinite dimensional extensions of the metric characterization of stability (see [MFK], [DK]).

The same approach gives in the case of oriented pairs the following differential geometric interpretation of stability [OT5]: 
Let $E$ be a differentiable rank 2 vector bundle over a compact Kähler manifold $(X, g), \mathcal{L}$ a holomorphic structure in $L:=\operatorname{det}(E)$ and $l$ a fixed Hermitian metric in $L$.

Theorem 2.2.1 An holomorphic pair $(\mathcal{E}, \varphi)$ of type $(E, \mathcal{L})$ with $\operatorname{rk}(\mathcal{E})=2$ is polystable iff $\mathcal{E}$ admits a Hermitian metric $h$ with $\operatorname{det} h=l$ which solves the following projective vortex equation:

$$
i \Lambda_{g} F_{h}^{0}+\frac{1}{2}\left(\varphi \bar{\varphi}^{h}\right)_{0}=0 .
$$

If $(\mathcal{E}, \varphi)$ is stable, then the metric $h$ is unique.

Remark 2.2.2 With an appropriate definition of (poly)stability of oriented pairs [OST], the theorem holds for arbitrary rank $r$.

Denote by $\lambda \in \mathcal{A}(L)$ the the Chern connection of $\mathcal{L}$ associated with the metric $l$. Let $\overline{\mathcal{A}}_{\bar{\partial}_{\lambda}}$ be the space of semiconnections in $E$ which induce the fixed semiconnection $\hat{\partial}_{\lambda}$ in $L$.

Fix a Hermitian metric $H$ in $E$ with $\operatorname{det} H=l$ and denote by $\mathcal{A}_{\lambda}$ the space of unitary connections in $E$ with induce the fixed connection $\lambda$ in $L$. There is an obvious identification $\mathcal{A}_{\lambda} \stackrel{\sim}{\longrightarrow} \overline{\mathcal{A}}_{\bar{\partial}_{\lambda}}, C \longmapsto \bar{\partial}_{C}$ which endows the affine space $\mathcal{A}_{\lambda}$ with a complex structure compatible with the standard $L^{2}$ euclidean structure. Therefore, after suitable Sobolev completions, the product $\mathcal{A}_{\lambda} \times A^{0}(E)=\overline{\mathcal{A}}_{\bar{\partial}_{\lambda}} \times A^{0}(E)$ becomes a Hilbert Kähler manifold. Let $\mathcal{G}_{0}:=$ $\Gamma(X, S U(E))$ be the gauge group of unitary automorphisms of determinant 1 in $(E, H)$ and let $\mathcal{G}_{0}^{\mathbb{C}}:=\Gamma(X, S L(E))$ be its complexification.

Remark 2.2.3 The map $m: \mathcal{A}_{\lambda} \times A^{0}(E) \longrightarrow A^{0}(s u(E))$ defined by

$$
m(C, \varphi)=\Lambda_{g} F_{C}^{0}-\frac{i}{2}\left(\varphi \bar{\varphi}^{H}\right)_{0}
$$

is a moment map for the $\mathcal{G}_{0}$-action in the Kähler manifold $\mathcal{A}_{\lambda} \times A^{0}(E)$

If $\mathcal{E}$ is a holomorphic structure in $E$ with $\operatorname{det} \mathcal{E}=\mathcal{L}$ we denote by $C_{\mathcal{E}} \in \mathcal{A}_{\lambda}$ the Chern connection defined be $\mathcal{E}$ and the fixed metric $H$. The map $(\mathcal{E}, \varphi) \longmapsto$ $\left(C_{\mathcal{E}}, \varphi\right)$ identifies the set of oriented pairs of type $(E, \mathcal{L})$ with the subspace $Z(j)$ of the affine space $\mathcal{A}_{\lambda} \times A^{0}(E)$ which is cut-out by the integrability condition

$$
j(C, \varphi):=\left(F_{C}^{02}, \bar{\partial}_{C} \varphi\right)=0
$$

Definition 2.2.4 A pair $(C, \varphi) \in \mathcal{A}_{\lambda} \times A^{0}(E)$ will be called irreducible if any $C$-parallel endomorphism $f \in A^{0}(s u(E))$ with $f(\varphi)=0$ vanishes. 
This notion of (ir)reducibility must not be confused with that one introduced in section 1.2.3, which depends on the choice of an admissible pair. For instance, irreducible pairs can be abelian.

The theorem above can now be reformulated as follows:

Proposition 2.2.5 An oriented pair $(\mathcal{E}, \varphi)$ of type $(E, \mathcal{L})$ is polystable if and only if the complex orbit $\mathcal{G}_{0}^{\mathbb{C}} \cdot\left(C_{\mathcal{E}}, \varphi\right) \subset Z(j)$ intersects the vanishing locus $Z(m)$ of the moment map $m .(\mathcal{E}, \varphi)$ is stable if and only if it is polystable and $\left(C_{\mathcal{E}}, \varphi\right)$ is irreducible.

It can be easily seen that the intersection $\left[\mathcal{G}_{0}^{\mathbb{C}} \cdot\left(C_{\mathcal{E}}, \varphi\right)\right] \cap Z(m)$ of a complex orbit with the vanishing locus of the moment map is either empty or coincides with a real orbit. Moreover, using the proposition above one can prove that the set $\overline{Z(j})^{s t}$ of stable oriented pairs is an open subset of the set $Z(j)^{s}$ of simple oriented pairs. The quotient $Z(j)^{s} / \mathcal{G}_{0}^{\mathbb{C}}$ can be identified with the moduli space $\mathcal{M}^{s}(E, \mathcal{L})$ of simple oriented pairs of type $(E, \mathcal{L})$. The open subspace $\mathcal{M}^{s t}(E, \mathcal{L}):=Z(j)^{s t} / \mathcal{G}_{0}^{\mathbb{C}} \subset \mathcal{M}^{s}(E, \mathcal{L})$ will be called the moduli space of stable oriented pairs, and comes with a natural structure of a Hausdorff complex space.

The same methods as in [DK], [LT], [OT1] give finally the following

Theorem 2.2.6 The identification map $(C, \varphi) \longmapsto\left(\bar{\partial}_{C}, \varphi\right)$ induces an isomorphism of real analytic spaces $Z(j, m)^{i r} / \mathcal{G}_{0} \stackrel{\sim}{\rightarrow} Z(j)^{\text {st }} / \mathcal{G}_{0}^{\mathbb{C}}=\mathcal{M}^{\text {st }}(E, \mathcal{L})$, where $Z(j, m)^{\text {ir }}$ denotes the locally closed subspace consisting of irreducible oriented pairs solving the equations $j(C, \varphi)=0, m(C, \varphi)=0$.

\subsection{Decoupling the $P U(2)$-monopole equations}

Let $(X, g)$ be a Kähler surface and let $P^{\text {can }} \longrightarrow P_{g}$ be the associated canonical $\operatorname{Spin}^{c}(4)$-structure whose spinor bundles are $\Sigma^{+}=\Lambda^{00} \oplus \Lambda^{02}, \Sigma^{-}=\overline{\Lambda^{01} \text {. By }}$ Propositions 1.1.11, 1.1.7 it follows that the data of a $\operatorname{Spin}^{U(2)}(4)$-structure in $(X, g)$ is equivalent to the data of a Hermitian 2-bundle $E$. The bundles associated with the $\operatorname{Spin}^{U(2)}(4)$-structure $\sigma: P^{u} \longrightarrow P_{g}$ corresponding to $E$ are:

$\operatorname{det}\left(P^{u}\right)=\operatorname{det} E \otimes K_{X}, \bar{\delta}\left(P^{u}\right)=P_{E} / S^{1}$,

$\Sigma^{ \pm}\left(P^{u}\right)=\Sigma^{ \pm} \otimes E^{\vee} \otimes \operatorname{det}\left(P^{u}\right)=\Sigma^{ \pm} \otimes E \otimes K_{X} ; \quad \Sigma^{+}\left(P^{u}\right)=E \otimes K_{X} \oplus E$.

Suppose that $\operatorname{det}\left(P^{u}\right) \in N S(X)$ and fix an integrable connection $a \in \mathcal{A}\left(\operatorname{det}\left(P^{u}\right)\right)$. Denote by $c \in \mathcal{A}\left(K_{X}\right)$ the Chern connection in $K_{X}$, by $\lambda:=a \otimes \bar{c}$ the induced connection in $\operatorname{det}(E)=\operatorname{det}\left(P^{u}\right) \otimes \bar{K}_{X}$ and by $\mathcal{L}$ the corresponding holomorphic structure in this line bundle. Identify the affine space $\mathcal{A}\left(\bar{\delta}\left(P^{u}\right)\right)$ with $\mathcal{A}_{\lambda \otimes c} \otimes^{2}\left(E \otimes K_{X}\right)$ and the space of spinors $A^{0}\left(\Sigma^{+}\left(P^{u}\right)\right)$ with the direct sum $A^{0}\left(E \otimes K_{X}\right) \oplus A^{0}(E)=A^{0}\left(E \otimes K_{X}\right) \oplus A^{02}\left(E \otimes K_{X}\right)$. The same computations as in Proposition 4.1 [OT5] gives the following decoupling theorem: 
Theorem 2.3.1 A pair

$$
(C, \varphi+\alpha) \in \mathcal{A}_{\lambda \otimes c^{\otimes 2}}\left(E \otimes K_{X}\right) \times\left(A^{0}\left(E \otimes K_{X}\right) \oplus A^{02}\left(E \otimes K_{X}\right)\right)
$$

solve the $P U(2)$-monopole equations $S W_{a}^{\sigma}$ if and only if the connection $C$ is integrable and one of the following conditions is fulfilled:

$$
\begin{aligned}
& \text { 1) } \alpha=0, \bar{\partial}_{C} \varphi=0 \quad \text { and } \quad i \Lambda_{g} F_{C}^{0}+\frac{1}{2}(\varphi \bar{\varphi})_{0}=0, \\
& \text { 2) } \varphi=0, \partial_{C} \alpha=0 \text { and } i \Lambda_{g} F_{C}^{0}-\frac{1}{2} *(\alpha \wedge \bar{\alpha})_{0}=0
\end{aligned}
$$

Using Theorem 2.2.5 we get

Remark 2.3.2 The moduli space $\left(\mathcal{M}_{a}^{\sigma}\right)_{\alpha=0}^{i r}$ of irreducible solutions of type 1) can be identified with the moduli space $\mathcal{M}^{\text {st }}\left(E \otimes K_{X}, \mathcal{L} \otimes \mathcal{K}_{X}^{\otimes 2}\right)$.

The moduli space $\left(\mathcal{M}_{a}^{\sigma}\right)_{\varphi=0}^{i r}$ of irreducible solutions of type 2) can be identified with the moduli space $\mathcal{M}^{s t}\left(E^{\vee}, \mathcal{L}^{\vee}\right)$ via the map $(C, \alpha) \longmapsto(\bar{C} \otimes c, \bar{\alpha})$.

Concluding, we get the following simple description of the moduli space $\mathcal{M}_{a}^{\sigma}$ in terms of moduli spaces of stable oriented pairs.

Corollary 2.3.3 Suppose that the $\operatorname{Spin}^{U(2)}(4)$-structure $\sigma: P^{u} \longrightarrow P_{g}$ is associated to the pair $\left(P^{\text {can }} \longrightarrow P_{g}, E\right)$, where $P^{\text {can }} \longrightarrow P_{g}$ is the canonical Spinc $^{c}(4)$-structure of the Kähler surface $(X, g)$ and $E$ is a Hermitian rank 2 bundle. Let $a \in \mathcal{A}\left(\operatorname{det}\left(P^{u}\right)\right)$ be an integrable connection and $\mathcal{L}$ the holomorphic structure in $\operatorname{det} E=\operatorname{det}\left(P^{u}\right) \otimes K_{X}^{\vee}$ defined by a and the Chern connection in $K_{X}$. Then the moduli space $\mathcal{M}_{a}^{\sigma}$ decomposes as a union of two Zariski closed subspaces

$$
\mathcal{M}_{a}^{\sigma}=\left(\mathcal{M}_{a}^{\sigma}\right)_{\alpha=0} \bigcup\left(\mathcal{M}_{a}^{\sigma}\right)_{\varphi=0}
$$

which intersect along the Donaldson moduli space $\mathcal{D}\left(\delta\left(P^{u}\right)\right) \subset \mathcal{M}_{a}^{\sigma}$ (see Remark 1.2.8). There are canonical real analytic isomorphisms

$$
\left(\mathcal{M}_{a}^{\sigma}\right)_{\alpha=0}^{i r} \simeq \mathcal{M}^{s t}\left(E \otimes K_{X}, \mathcal{L} \otimes \mathcal{K}_{X}^{\otimes 2}\right), \quad\left(\mathcal{M}_{a}^{\sigma}\right)_{\varphi=0}^{i r}=\mathcal{M}^{s t}\left(E^{\vee}, \mathcal{L}^{\vee}\right)
$$

Using Remark 1.2.7, we recover the main result (Theorem 7.3) in [OT5] stated for quaternionic monopoles.

Example: (R. Plantiko) On $\mathbb{P}^{2}$ endowed with the standard Fubini-Study metric $g$ consider the $\operatorname{Spin}^{U(2)}(4)$-structure $P^{u} \longrightarrow P_{g}$ with $c_{1}\left(\operatorname{det}\left(P^{u}\right)\right)=4$, $p_{1}\left(\bar{\delta}\left(P^{u}\right)\right)=-3$. It is easy to see that this $\operatorname{Spin}^{U(2)}(4)$-structure is associated with the pair $\left(P^{\text {can }} \longrightarrow P_{g}, E\right)$, where $E$ is a $U(2)$-bundle with $c_{2}(E)=13$, $c_{1}(E)=7$. Therefore $E \otimes K$ has $c_{1}(E \otimes K)=1, c_{2}(E \otimes K)=1$. 
Using Remark 2.1.4 it is easy to see that any stable oriented pair $(\mathcal{F}, \varphi)$ of type $(E \otimes K, \mathcal{O}(1))$ with $\varphi \neq 0$ fits in an exact sequence of the form

$$
0 \longrightarrow \mathcal{O} \stackrel{\varphi}{\longrightarrow} \longrightarrow \mathcal{O}(1) \otimes J_{z_{\varphi}} \longrightarrow 0,
$$

where $z_{\varphi} \in \mathbb{P}^{2}, c \in \mathbb{C}$ and $\mathcal{F}=\mathcal{T}_{\mathbb{P}^{2}}(-1)$ is the unique stable bundle with $c_{1}=c_{2}=1$. Moreover, two oriented pairs $(\mathcal{F}, \varphi),\left(\mathcal{F}, \varphi^{\prime}\right)$ define the same point in the moduli space of stable oriented pairs of type $(E \otimes K, \mathcal{O}(1))$ if and only if $\varphi^{\prime}= \pm \varphi$. Therefore

$$
\mathcal{M}^{s t}(E \otimes K, \mathcal{O}(1))=\left.H^{0}(\mathcal{F})\right|_{ \pm \mathrm{id}} \simeq \mathbb{C}^{3} / \pm \mathrm{id}
$$

Studying the local models of the moduli space one can check that the above identification is a complex analytic isomorphism.

On the other hand every polystable oriented pair of type $(E \otimes K, \mathcal{O}(1))$ is stable and there is no polystable oriented pair of type $\left(E^{\vee}, \mathcal{O}(-7)\right)$. This shows that

$$
\mathcal{M}_{a}^{\sigma} \simeq \mathbb{C}^{3} / \pm \mathrm{id}
$$

for every integrable connection $a \in \mathcal{A}\left(\operatorname{det}\left(P^{u}\right)\right)$. The quotient $\mathbb{C}^{3} / \pm$ id has a natural compactification $\mathcal{C}:=\mathbb{P}^{3} /\langle\iota\rangle$, where $\iota$ is the involution

$$
\left[x_{0}, x_{1}, x_{2}, x_{3}\right] \longmapsto\left[x_{0},-x_{1},-x_{2},-x_{3}\right] .
$$

$\mathcal{C}$ can be identified with cone over the image of $\mathbb{P}^{2}$ under the Veronese map $v_{2}: \mathbb{P}^{2} \longrightarrow \mathbb{P}^{5}$. This compactification coincides with the Uhlenbeck compactification of the moduli space [T1], [T2]. Let now $\sigma^{\prime}: P^{\prime u} \longrightarrow P_{g}$ be the $\operatorname{Spin}^{U(2)}(4)$ structure in $\mathbb{P}^{2}$ with $\operatorname{det}\left(P^{\prime u}\right)=\operatorname{det}\left(P^{u}\right), p_{1}\left(\delta\left(P^{\prime u}\right)\right)=+1$. It is easy to see by the same method that $\mathcal{M}_{a}^{\sigma^{\prime}}$ consists of only one point, which is the abelian solution associated with the stable oriented pair $\left(\mathcal{O} \oplus \mathcal{O}(1), i_{\mathcal{O}}\right)$. Via the isomorphism explained in Proposition 1.2.15, $\mathcal{M}_{a}^{\sigma^{\prime}}$ corresponds to the moduli space of solutions of the (abelian) twisted Seiberg-Witten equations associated with the canonical $\operatorname{Spin}^{c}(4)$-structure and the positive chamber (see [OT6]). Therefore

Proposition 2.3.4 The Uhlenbeck compactification of the moduli space $\mathcal{M}_{a}^{\sigma}$ can be identified with the cone $\mathcal{C}$ over the image of $\mathbb{P}^{2}$ under the Veronese map $v_{2}$. The vertex of the cone corresponds to the unique Donaldson point. The base of the cone corresponds to the space $\mathcal{M}_{a}^{\sigma^{\prime}} \times \mathbb{P}^{2}$ of ideal solutions concentrated in one point. The moduli space $\mathcal{M}_{a}^{\sigma^{\prime}}$ consists of only one abelian point. 


\section{References}

[AHS] Atiyah M., Hitchin N. J., Singer I. M.: Selfduality in four-dimensional Riemannian geometry, Proc. R. Lond. A. 362, 425-461 (1978)

[B] Bradlow, S. B.: Special metrics and stability for holomorphic bundles with global sections, J. Diff. Geom. 33, 169-214 (1991)

[D] Donaldson, S.: Anti-self-dual Yang-Mills connections over complex algebraic surfaces and stable vector bundles, Proc. London Math. Soc. 3, 1-26 (1985)

[DK] Donaldson, S.; Kronheimer, P.B.: The Geometry of four-manifolds, Oxford Science Publications 1990

[FU] Freed D. S. ; Uhlenbeck, K.: Instantons and Four-Manifolds. SpringerVerlag 1984

[GS] Guillemin, V.; Sternberg, S.: Birational equivalence in the symplectic category, Inv. math. 97, 485-522 (1989)

[HH] Hirzebruch, F.; Hopf, H.: Felder von Flächenelementen in 4-dimensionalen 4-Mannigfaltigkeiten, Math. Ann. 136 (1958)

[H] Hitchin, N.: Harmonic spinors, Adv. in Math. 14, 1-55 (1974)

[HKLR] Hitchin, N.; Karlhede, A.; Lindström, U.; Roček, M.: Hyperkähler metrics and supersymmetry, Commun. Math. Phys. (108), 535-589 (1987)

[K] Kobayashi, S.: Differential geometry of complex vector bundles, Princeton University Press 1987

[KM] Kronheimer, P.; Mrowka, T.: The genus of embedded surfaces in the projective plane, Math. Res. Letters 1, 797-808 (1994)

[LL] Li, T.; Liu, A.: General wall crossing formula, Math. Res. Lett. 2, 797-810 (1995).

[LM] Labastida, J. M. F.; Marino, M.: Non-abelian monopoles on four manifolds, Preprint, Departamento de Fisica de Particulas, Santiago de Compostela, April (1995)

[La] Larsen, R.: Functional analysis, an introduction, Marcel Dekker, Inc., New York, 1973

[LMi] Lawson, H. B. Jr.; Michelson, M. L.: Spin Geometry, Princeton University Press, New Jersey, 1989

[LT] Lübke, M.; Teleman, A.: The Kobayashi-Hitchin correspondence, World Scientific Publishing Co. 1995

[M] Miyajima, K.: Kuranishi families of vector bundles and algebraic description of the moduli space of Einstein-Hermitian connections, Publ. R.I.M.S. Kyoto Univ. 25, 301-320 (1989)

[MFK] Mumford, D,; Fogarty, J.; Kirwan, F.: Geometric invariant theory, Springer Verlag, 1994

[OST] Okonek, Ch.; Schmitt, A.; Teleman, A.: Master spaces for stable pairs, Preprint, alg-geom/9607015

[OT1] Okonek, Ch.; Teleman, A.: The Coupled Seiberg-Witten Equations, Vortices, and Moduli Spaces of Stable Pairs, Int. J. Math. Vol. 6, No. 6, 893-910 
(1995)

[OT2] Okonek, Ch.; Teleman, A.: Les invariants de Seiberg-Witten et la conjecture de Van De Ven, Comptes Rendus Acad. Sci. Paris, t. 321, Série I, 457-461 (1995)

[OT3] Okonek, Ch.; Teleman, A.: Seiberg-Witten invariants and rationality of complex surfaces, Math. Z., to appear

[OT4] Okonek, Ch.; Teleman, A.: Quaternionic monopoles, Comptes Rendus Acad. Sci. Paris, t. 321, Série I, 601-606 (1995)

[OT5] Ch, Okonek.; Teleman, A.: Quaternionic monopoles, Commun. Math. Phys., Vol. 180, Nr. 2, 363-388, (1996)

[OT6] Ch, Okonek.; Teleman, A.: Seiberg-Witten invariants for manifolds with $b_{+}=1$, and the universal wall crossing formula, Int. J. Math., to appear

[PT1] Pidstrigach, V.; Tyurin, A.: Invariants of the smooth structure of an algebraic surface arising from the Dirac operator, Russian Acad. Izv. Math., Vol. 40, No. 2, 267-351 (1993)

[PT2] Pidstrigach, V.; Tyurin, A.: Localisation of the Donaldson invariants along the Seiberg-Witten classes, Russian Acad. Izv. , to appear

[T1] Teleman, A. :Non-abelian Seiberg-Witten theory, Habilitationsschrift, Universität Zürich, 1996

[T2] Teleman, A. :Moduli spaces of PU(2)-monopoles, Preprint, Universität Zürich, 1996

[W] Witten, E.: Monopoles and four-manifolds, Math. Res. Letters 1, 769-796 (1994)

Author's address : Institut für Mathematik, Universität Zürich, Winterthurerstr. 190, CH-8057 Zürich, e-mail: teleman@math.unizh.ch

and Department of Mathematics, University of Bucharest. 